\title{
Calculating quenching weights
}

\author{
Carlos A. Salgado and Urs Achim Wiedemann \\ Theory Division, CERN, CH-1211 Geneva 23, Switzerland \\ (Received 20 February 2003; published 10 July 2003)
}

\begin{abstract}
We calculate the probability ("quenching weight") that a hard parton radiates an additional energy fraction $\Delta E$ due to scattering in spatially extended QCD matter. This study is based on an exact treatment of a finite in-medium path length; it includes the case of a dynamically expanding medium, and it extends to the angular dependence of the medium-induced gluon radiation pattern. All calculations are done in the multiple soft scattering approximation [Baier-Dokshitzer-Mueller-Peigné-Schiff-Zakharov (BDMPSZ) formalism] and in the single hard scattering approximation $[N=1$ opacity approximation]. By comparison, we establish a simple relation between the transport coefficient, Debye screening mass and opacity, for which both approximations lead to comparable results. Together with this paper, a CPU-inexpensive numerical subroutine for calculating quenching weights is provided electronically. To illustrate its applications, we discuss the suppression of hadronic transverse momentum spectra in nucleus-nucleus collisions. Remarkably, the kinematic constraint resulting from finite in-medium path lengths reduces significantly the $p_{\perp}$ dependence of the nuclear modification factor, thus leading to consistency with the data measured at the BNL Relativistic Heavy Ion Collider.
\end{abstract}

DOI: 10.1103/PhysRevD.68.014008

PACS number(s): 12.38.Mh, 24.85.+p, 25.75.-q

\section{INTRODUCTION}

Hard partons produced in nucleus-nucleus collisions at the BNL Relativistic Heavy Ion Collider (RHIC) and CERN Large Hadron Collider (LHC) propagate through highly excited matter before hadronizing in the vacuum. The resulting medium dependence of parton fragmentation is expected to affect hadronic observables. This is of twofold interest. First, it provides a novel test of the space-time evolution of the perturbative parton shower. Second, the modification of hadronic observables due to the spatially extended, hot and dense QCD matter allows us to characterize the properties of the transient state produced in the collision.

Gluon emission off highly virtual hard partons is an essential component in the standard description of parton fragmentation in elementary processes. This effect degrades the energy of the leading parton. Recently, it has been proposed [1] that in the presence of a spatially extended medium, the additional medium-induced energy degradation of the leading parton can be described by a probability $P(\Delta E)$, the so-called quenching weight, which is obtained from a probabilistic iteration of the medium-modified elementary splitting processes $q \rightarrow q g$ and $g \rightarrow g g$. The main purpose of the present work is to calculate and compare this quenching weight for different approximations of the medium-modified splitting process, to make the results for $P(\Delta E)$ available as a numerical subroutine, and to illustrate the use of this subroutine with some applications.

We start from recent calculations [2-5] of the modification of the elementary splitting processes $q \rightarrow q g$ and $g$ $\rightarrow g g$ due to multiple scattering. These results go under the name medium-induced gluon radiation. They present limiting cases of a unique path-integral expression given in Eq. (2.1) below. Technically, they collect all terms to leading order in nuclear enhanced modifications $O\left(\alpha_{s} A^{1 / 3}\right)$, thus accounting for the leading additional interactions of the parton shower with the medium.

The paper is organized as follows. In Sec. II, we compare the medium-induced gluon energy distribution radiated off a hard parton in two limits which emphasize the role of multiple soft and single hard medium-induced scatterings, respectively. In Sec. III we give results for the quenching weights corresponding to these limits. These quenching weights can be calculated with the numerical subroutine accompanying this paper. In Sec. IV, we extend these calculations to the case of an expanding medium, and in Sec. V, we discuss the extension to radiation within a finite cone. As application, we calculate in Sec. VI in two different approaches the suppression of hadronic transverse momentum spectra and we compare our results to the nuclear modification factor measured in $\mathrm{Au}-\mathrm{Au}$ collisions at the Relativistic Heavy Ion Collider (RHIC).

\section{MEDIUM-INDUCED GLUON RADIATION FROM A STATIC MEDIUM}

The inclusive energy distribution of gluon radiation off an in-medium produced parton takes the form $[4,6,7]$

$$
\begin{aligned}
\omega \frac{d I}{d \omega}= & \frac{\alpha_{s} C_{R}}{(2 \pi)^{2} \omega^{2}} 2 \operatorname{Re} \int_{\xi_{0}}^{\infty} d y_{l} \int_{y_{l}}^{\infty} d \bar{y}_{l} \int d \mathbf{u} \int_{0}^{\chi \omega} d \mathbf{k}_{\perp} e^{-i \mathbf{k}_{\perp} \cdot \mathbf{u}} \\
& \times e^{-(1 / 2) \int_{y_{l}}^{\infty} d \xi n(\xi) \sigma(\mathbf{u})} \frac{\partial}{\partial \mathbf{y}} \cdot \frac{\partial}{\partial \mathbf{u}} \int_{\mathbf{y}=0=\mathbf{r}\left(y_{l}\right)}^{\mathbf{u}=\mathbf{r}\left(\bar{y}_{l}\right)} \mathcal{D} \mathbf{r} \\
& \times \exp \left[i \int_{y_{l}}^{y_{l}} d \xi \frac{\omega}{2}\left(\dot{\mathbf{r}}^{2}-\frac{n(\xi) \sigma(\mathbf{r})}{i \omega}\right)\right]
\end{aligned}
$$

Here, $\mathbf{k}_{\perp}$ denotes the transverse momentum of the emitted gluon. The limit $k_{\perp}=\left|\mathbf{k}_{\perp}\right|<\chi \omega$ on the transverse phase space allows to discuss gluon emission into a finite opening angle $\Theta, \chi=\sin \Theta$. For the full angular integrated quantity, $\chi=1$.

The radiation of hard quarks or gluons differs by the $\mathrm{Ca}$ simir factor $C_{R}=C_{F}$ or $C_{A}$, respectively. Numerical results are for fixed coupling constant $\alpha_{s}=1 / 3$, except where stated 
otherwise. The properties of the medium enter Eq. (2.1) in terms of the product of the time-dependent density $n(\xi)$ of scattering centers times the strength of a single elastic scattering $\sigma(\mathbf{r})$. This dipole cross section $\sigma(\mathbf{r})$ is given in terms of the elastic high-energy cross section $|a(\mathbf{q})|^{2}$ of a single scatterer in the color octet representation:

$$
\sigma(\mathbf{r})=2 \int \frac{d \mathbf{q}}{(2 \pi)^{2}}|a(\mathbf{q})|^{2}\left(1-e^{i \mathbf{q} \cdot \mathbf{r}}\right) .
$$

Equation (2.1) is derived by resumming the multiple scattering Feynman diagrams for the gluon radiation amplitude to leading order $O\left(\alpha_{s} A^{1 / 3}\right)$ in the norm and phase [4]. The twodimensional transverse coordinates $\mathbf{u}, \mathbf{y}$, and $\mathbf{r}$ emerge in the derivation of Eq. (2.1) as distances between the positions of projectile components in the amplitude and complex conjugate amplitude. The longitudinal coordinates $y_{l}, \bar{y}_{l}$ integrate over the ordered longitudinal gluon emission points in amplitude and complex conjugate amplitude, which emerge in time-ordered perturbation theory. For longitudinal positions $\xi>\bar{y}_{l}$ for which a gluon is emitted in both amplitude and complex conjugate amplitude, the dipole cross section $\sigma(\mathbf{u})$ in Eq. (2.1) measures the impact parameter difference $\mathbf{u}$ between both gluons. By Fourier transformation, this difference $\mathbf{y}$ is related to the final transverse momentum $\mathbf{k}_{\perp}$ of the emitted gluon. For longitudinal positions $y_{l}<\xi<\bar{y}_{l}$ for which a gluon is emitted in the amplitude but not yet in the complex conjugate amplitude, the dipole cross section $\sigma(\mathbf{r})$ in Eq. (2.1) measures the impact parameter difference $\mathbf{r}$ between the emitted gluon and the position of the emitting quarkantiquark system in amplitude and complex conjugate amplitude. Technically, this results again in the cross section for a dipole with two color octet legs. In the following discussion, this space-time interpretation of the internal integration variables in Eq. (2.1) plays no role. An explicit derivation and more detailed discussion of Eq. (2.1) can be found in Ref. [4].

Theoretical uncertainties related to Eq. (2.1) have at least two origins. First, the derivation of Eq. (2.1) employs the high-energy approximation in which the medium acts as a collection of static scattering centers and the initial parton loses a small additional medium-induced amount of its total energy, $\Delta E \ll E$. Thus, Eq. (2.1) can be expected to be reliable for $\Delta E \ll E$ if the spectrum $\omega(d I / d \omega)$ is perturbatively hard (which will be established in Figs. 1 and 3 below). However, it is unknown so far, to what extent Eq. (2.1) receives corrections for parameter values for which $\Delta E$ $\sim O(E)$. Second, for dipole cross sections $\sigma(\mathbf{r})$ of general functional shape, the evaluation of the path integral in Eq. (2.1) requires a further approximation. We start our study of the energy distribution (2.1) for a static medium by comparing two approximations: the saddle-point approximation and the expansion of Eq. (2.1) to first order in the number of scattering centers. As explained below, these approximations may be viewed as extreme limiting cases since they focus on the multiple soft and single hard momentum transfer from the medium, respectively.

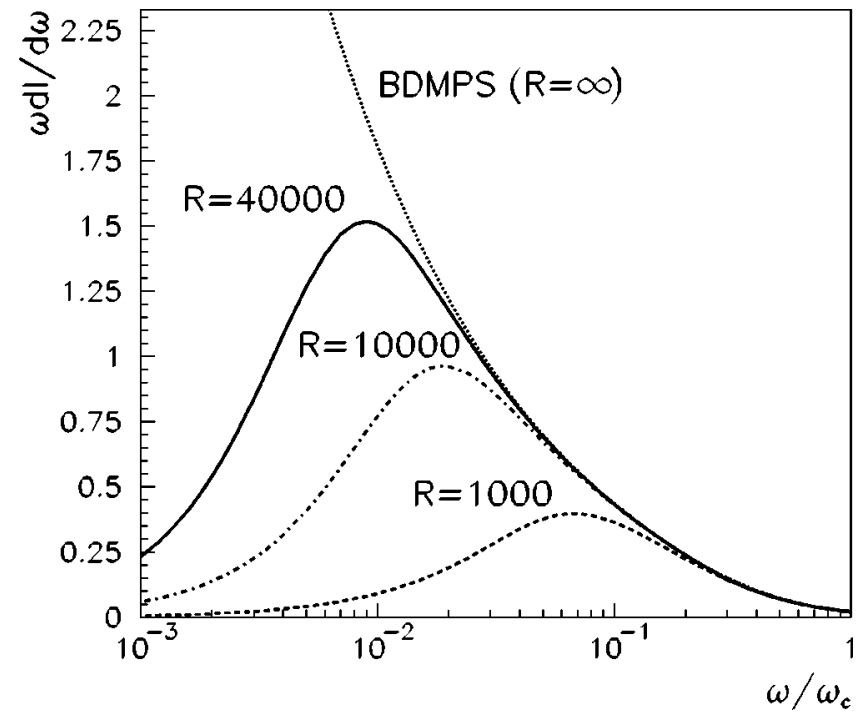

FIG. 1. The medium-induced gluon energy distribution $\omega(d I / d \omega)$ in the multiple soft scattering approximation for different values of the kinematic constraint $R=\omega_{c} L$.

\section{A. Multiple soft scattering approximation}

For arbitrary many soft scattering centers, the projectile performs a Brownian motion in transverse momentum. This dynamical limiting case can be studied in the saddle point approximation of the path integral (2.1), using $[3,8]$

$$
n(\xi) \sigma(\mathbf{r}) \simeq \frac{1}{2} \hat{q}(\xi) \mathbf{r}^{2} .
$$

Here, $\hat{q}(\xi)$ is the transport coefficient [9] which characterizes the medium-induced transverse momentum squared $\left\langle q_{\perp}^{2}\right\rangle_{\text {med }}$ transferred to the projectile per unit path length $\lambda$. For a static medium, the transport coefficient is time independent:

$$
\hat{q}=\frac{\left\langle q_{\perp}^{2}\right\rangle_{\mathrm{med}}}{\lambda} .
$$

In the approximation (2.3), the path integral in Eq. (2.1) is equivalent to that of a harmonic oscillator. The corresponding analytical expressions are summarized in Appendix A.

Qualitative arguments [10]. We consider a gluon in the hard parton wave function. This gluon is emitted due to multiple scattering if it picks up sufficient transverse momentum to decohere from the partonic projectile. For this, the average phase $\varphi$ accumulated by the gluon should be of order one:

$$
\varphi=\left\langle\frac{k_{\perp}^{2}}{2 \omega} \Delta z\right\rangle \sim \frac{\hat{q} L}{2 \omega} L=\frac{\omega_{c}}{\omega} .
$$

Thus, for a hard parton traversing a finite path length $L$ in the medium, the scale of the radiated energy distribution is set by the "characteristic gluon frequency"

$$
\omega_{c}=\frac{1}{2} \hat{q} L^{2} .
$$


For an estimate of the shape of the energy distribution, we consider the number $N_{\text {coh }}$ of scattering centers which add coherently in the gluon phase (2.5), $k_{\perp}^{2} \simeq N_{\text {coh }} \mu^{2}$. Based on expressions for the coherence time of the emitted gluon, $t_{\mathrm{coh}} \simeq \omega / k_{\perp}^{2} \simeq \sqrt{\omega / \hat{q}}$ and $N_{\mathrm{coh}}=t_{\mathrm{coh}} / \lambda=\sqrt{\omega / \mu^{2} \lambda}$, one estimates, for the gluon energy spectrum per unit path length,

$$
\omega \frac{d I}{d \omega d z} \simeq \frac{1}{N_{\mathrm{coh}}} \omega \frac{d I^{1 \text { scatt }}}{d \omega d z} \simeq \frac{\alpha_{s}}{t_{\mathrm{coh}}} \simeq \alpha_{s} \sqrt{\frac{\hat{q}}{\omega}} .
$$

This $1 / \sqrt{\omega}$-energy dependence of the medium-induced nonAbelian gluon energy spectrum is expected for sufficiently small $\omega<\omega_{c}$.

Quantitative analysis. The gluon energy distribution (2.1) depends not only on $\omega_{c}$, but also on the constraint $k_{\perp}$ $<\chi \omega$ on the transverse momentum phase space of the emitted gluon. This enters the calculation via the dimensionless kinematic constraint [11]

$$
R_{\chi}=\frac{1}{2} \hat{q} \chi^{2} L^{3}, \quad R \equiv R_{\chi=1}=\omega_{c} L .
$$

This constraint is neglected in the argument leading to the $1 / \sqrt{\omega}$-energy dependence of Eq. (2.7). In the following sections, we limit the discussion to angular fully integrated quantities for which $\chi=1$. The only exception will be the discussion of the angular $\Theta$ dependence of $\omega(d I / d \omega)$ in Sec. V, where we use $\chi=\sin \Theta$.

The limit $R \rightarrow \infty$ which removes the kinematic constraint from Eq. (2.1) is either realized by extending the $k_{\perp}$ integration ad hoc to infinity. Alternatively, $R \rightarrow \infty$ can be viewed as the limit of infinite in-medium path length since it corresponds to $L \rightarrow \infty$ for $\chi$ and $\omega_{c}$ fixed. In Appendix A, we derive the $R \rightarrow \infty$ limit of the energy distribution (2.1),

$$
\lim _{R \rightarrow \infty} \omega \frac{d I}{d \omega}=\frac{2 \alpha_{s} C_{R}}{\pi} \ln \left|\cos \left[(1+i) \sqrt{\frac{\omega_{c}}{2 \omega}}\right]\right| .
$$

This coincides with the result of Baier, Dokshitzer, Mueller, Peigné, and Schiff [9]. As expected from the estimates in Eqs. (2.5) and (2.7), it shows a characteristic $1 / \sqrt{\omega}$-energy dependence for small $\omega$ which is suppressed above the characteristic gluon frequency $\omega_{c}[1]$ :

$$
\lim _{R \rightarrow \infty} \omega \frac{d I}{d \omega} \simeq \frac{2 \alpha_{s} C_{R}}{\pi} \begin{cases}\sqrt{\frac{\omega_{c}}{2 \omega}} & \text { for } \omega<\omega_{c}, \\ \frac{1}{12}\left(\frac{\omega_{c}}{\omega}\right)^{2} & \text { for } \omega>\omega_{c} .\end{cases}
$$

The average parton energy loss is the zeroth moment of this energy distribution

$$
\langle\Delta E\rangle_{R \rightarrow \infty}=\lim _{R \rightarrow \infty} \int_{0}^{\infty} d \omega \omega \frac{d I}{d \omega}=\frac{\alpha_{s} C_{R}}{2} \omega_{c} .
$$

This is the well-known $L^{2}$ dependence of the average energy loss $[2,9,12]$. Due to the steep fall-off at large $\omega$, the $\omega$ integral in Eq. (2.11) is dominated by the region $\omega$ $<\omega_{c} / \sqrt{2}$.

We have evaluated numerically the energy distribution (2.1) for finite values of the density parameter $R$. As seen in Fig. 1, the distribution approaches for any value of $R$ the Baier-Dokshitzer-Mueller-Peigné-Schiff (BDMPS) limit (2.9) at sufficiently large gluon energy. Below a critical gluon energy $\hat{\omega}$, however, the finite size gluon spectrum is depleted in comparison to the BDMPS limit. To understand this effect, we consider the characteristic angle $\Theta_{c}$ at which medium-induced gluons are radiated on average

$$
\Theta_{c}^{2} \simeq \frac{k_{\perp}^{2}}{\omega^{2}} \simeq \frac{\sqrt{\omega \hat{q}}}{\omega^{2}} \simeq\left(\frac{\omega}{\omega_{c}}\right)^{-3 / 2} \frac{1}{R} .
$$

For $\Theta_{c} \sim 1$, the emitted gluons are sensitive to the kinematic constraint since $k_{\perp} \sim O(\omega)$. The condition $\Theta_{c} \sim 1$ thus provides an estimate for the gluon energy $\hat{\omega}$ below which the energy distribution is cut off:

$$
\frac{\hat{\omega}}{\omega_{c}} \propto\left(\frac{1}{R}\right)^{2 / 3} .
$$

The position of the maximum of $\omega(d I / d \omega)$ as a function of $R$ is consistent with this dependence on $\hat{\omega}$, see Fig. 1. In general, gluon radiation at small energies corresponds to gluon radiation at large angle and is depleted as soon as the finite size of the transverse momentum phase space becomes relevant. This suppression of the nonperturbative small- $\omega$ contributions helps to make the calculation of mediuminduced energy loss perturbatively stable.

The gluon energy distribution (2.1) also determines the multiplicity $N(\omega)$ of gluons emitted with energies larger than $\omega$

$$
N(\omega) \equiv \int_{\omega}^{\infty} d \omega^{\prime} \frac{d I\left(\omega^{\prime}\right)}{d \omega^{\prime}} .
$$

In the absence of kinematic constraints, and for sufficiently small energies $\omega$, one finds from Eq. (2.10) that the total multiplicity diverges as $1 / \sqrt{\omega}$ [1],

$$
\lim _{R \rightarrow \infty} N(\omega)=\frac{2 \alpha_{s} C_{R}}{\pi} \sqrt{\frac{2 \omega_{c}}{\omega}} \text { for } \omega<\omega_{c} .
$$

However, realistic kinematic constraints on the transverse momentum phase space $(R<\infty)$ deplete the gluon energy distribution at small $\omega$ and ensure that the total gluon multiplicity $N(\omega=0)$ is finite, see Fig. 2.

For realistic kinematic constraints $R<10000$, the average additional total multiplicity is $N(\omega=0) \leqslant 3$. In comparison to the typically $\sim 5-10$ semihard partons which are the partonic final state of a $100 \mathrm{GeV}$ jet simulated in a parton shower, this additional multiplicity is not negligible. It sup- 


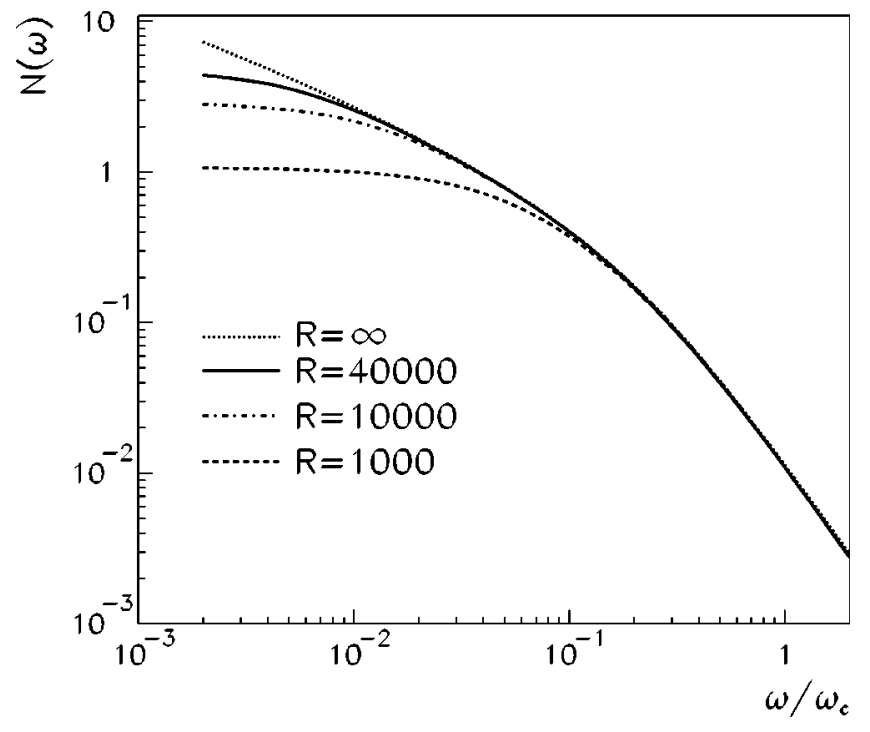

FIG. 2. The multiplicity of additional medium-induced gluons (2.14) radiated with energy larger than $\omega$. Calculation done in the multiple soft scattering approximation.

ports the naive expectation that the number of partons in the jet increases and softens with increasing transport coefficient or path length.

\section{B. Single hard scattering approximation}

In the previous subsection, we have studied the energy distribution (2.1) of medium-induced gluon radiation in the limit in which the partonic projectile performs a transverse Brownian motion due to multiple soft scattering. Now, we consider the opposite limiting case in which the radiation pattern results from an incoherent superposition of very few $n_{0} L$ single hard scattering processes positioned within path length $L$. This limit is obtained by expanding the integrand of the energy distribution $(2.1)$ in powers of $[n(\xi) \sigma(\mathbf{r})]^{N}$ up to first order $[4,5,13]$. Analytical expressions are given in Appendix B.

Qualitative arguments. We consider a hard partonic projectile which picks up a single transverse momentum $\mu$ by interacting with a single hard scatterer. An additional gluon of energy $\omega$ decoheres from the projectile wave function if its typical formation time $\bar{t}_{\text {coh }}=2 \omega / \mu^{2}$ is smaller than the typical distance $L$ between the production point of the parton and the position of the scatterer. The relevant phase is

$$
\gamma=\frac{L}{\bar{t}_{\mathrm{coh}}} \equiv \frac{\bar{\omega}_{c}}{\omega}
$$

which indicates a suppression of gluons with energy $\omega$ larger than the characteristic gluon energy

$$
\bar{\omega}_{c}=\frac{1}{2} \mu^{2} L
$$

The gluon energy spectrum per unit path length can be estimated in terms of the coherence time $\bar{t}_{\text {coh }}$ and of the average number $n_{0} L$ of scattering centers contributing incoherently

$$
\omega \frac{d I^{N=1}}{d \omega d z} \simeq\left(n_{0} L\right) \frac{\alpha_{s}}{\bar{t}_{\mathrm{coh}}} \simeq\left(n_{0} L\right) \alpha_{s} \frac{\mu^{2}}{\omega} .
$$

This is the typical $1 / \omega$ dependence of the non-Abelian gluon radiation spectrum in the absence of LPM-type destructive interference effects.

Quantitative analysis. We have calculated the first order in opacity $n_{0} L$ of the gluon energy distribution (2.1). To first order, the entire medium dependence comes from the interaction of the hard parton with a single static scattering center, multiplied by the number $n_{0} L=L / \lambda$ of scattering centers along the path. Modeling the single scatterer by a Yukawa potential with Debye screening mass $\mu$, we derive, in Appendix B,

$$
\begin{aligned}
\omega \frac{d I^{N=1}}{d \omega}= & 2 \frac{\alpha_{s} C_{R}}{\pi}\left(n_{0} L\right) \gamma \int_{0}^{\infty} d r \frac{r-\sin (r)}{r^{2}} \\
& \times\left(\frac{1}{r+\gamma}-\frac{1}{\sqrt{[(\bar{R} / 2 \gamma)+r+\gamma]^{2}-4 r \bar{R} / 2 \gamma}}\right) .
\end{aligned}
$$

This energy distribution depends on the phase factor $\gamma$ defined in Eq. (2.16), and on the kinematic constraint in transverse momentum phase space,

$$
\bar{R}_{\chi}=\frac{1}{2} \chi^{2} \mu^{2} L^{2}, \quad \bar{R} \equiv \bar{R}_{\chi=1}=\bar{\omega}_{c} L .
$$

In what follows, we work for $\chi=1$ except for the discussion of the angular dependence in Sec. V. In the limit in which the kinematic constraint is removed, the characteristic $1 / \omega$-energy dependence of the estimate (2.18) is recovered for sufficiently large gluon energies $\omega>\bar{\omega}_{c}$,

$$
\begin{aligned}
& \lim _{\bar{R} \rightarrow \infty} \omega \frac{d I^{N=1}}{d \omega}=2 \frac{\alpha_{s} C_{R}}{\pi}\left(n_{0} L\right) \gamma \int_{0}^{\infty} d r \frac{1}{r+\gamma} \frac{r-\sin (r)}{r^{2}} \\
& \simeq 2 \frac{\alpha_{s} C_{R}}{\pi}\left(n_{0} L\right)\left\{\begin{array}{cc}
\log \left[\frac{\bar{\omega}_{c}}{\omega}\right] & \text { for } \bar{\omega}_{c}>\omega, \\
\frac{\pi}{4} \frac{\bar{\omega}_{c}}{\omega} & \text { for } \bar{\omega}_{c}<\omega .
\end{array}\right.
\end{aligned}
$$

This limit agrees with the results of Gyulassy, Levai, and Vitev [13]. The average parton energy loss for a single hard scattering is dominated by contributions from the region $\omega$ $>\bar{\omega}_{c}[13,14]$, 


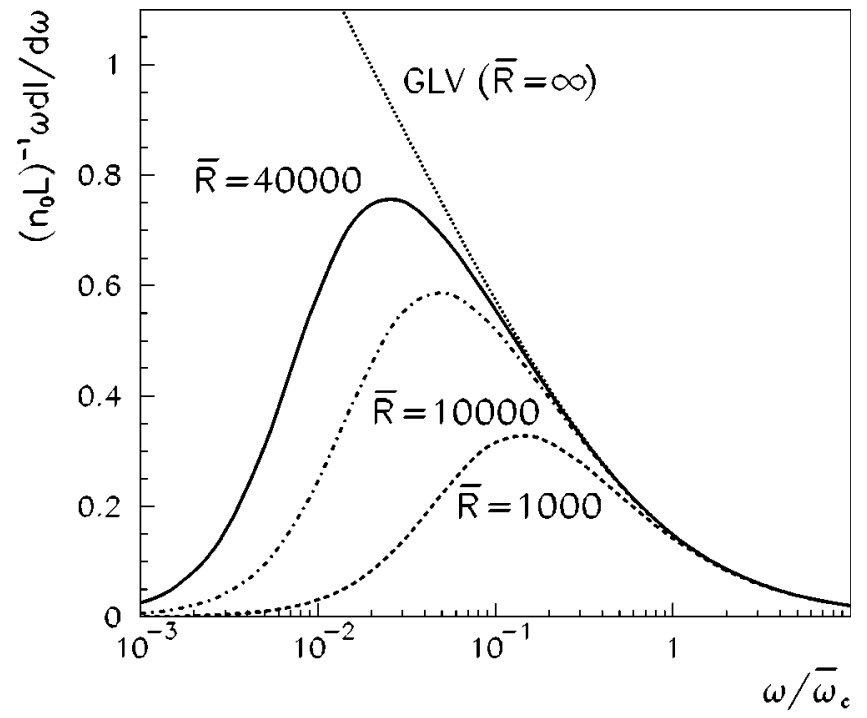

FIG. 3. The medium-induced gluon energy distribution $\omega(d I / d \omega)$ for a hard quark in the single hard scattering approximation, calculated for different values of the kinematic constraint $\bar{R}$.

$$
\begin{aligned}
\lim _{\bar{R} \rightarrow \infty}\langle\Delta E\rangle^{N=1} & =\lim _{\bar{R} \rightarrow \infty} \int d \omega \omega \frac{d I^{N=1}}{d \omega} \\
& \simeq \frac{\alpha_{s} C_{R}}{2}\left(n_{0} L\right) \bar{\omega}_{c} \log \left[E / \bar{\omega}_{c}\right] .
\end{aligned}
$$

It is logarithmically enhanced in comparison to the region $\omega<\bar{\omega}_{c}$ for which

$$
\lim _{\bar{R} \rightarrow \infty} \int_{0}^{\bar{\omega}_{c}} d \omega \omega \frac{d I^{N=1}}{d \omega} \simeq \frac{2 \alpha_{s} C_{R}}{\pi}\left(n_{0} L\right) \bar{\omega}_{c} .
$$

Remarkably, the average parton energy loss receives its dominant contribution from the hard region $\omega>\bar{\omega}_{c}$ in the opacity approximation (2.22) but from the soft region $\omega$ $<\omega_{c}$ in the multiple soft scattering approximation (2.11).

We have evaluated numerically the energy distribution (2.19) for finite values of the kinematic constraint $\bar{R}$. In close analogy to the multiple soft scattering approximation, the emission of soft gluons is suppressed in the opacity approximation due to the kinematic constraint $\bar{R}=\bar{\omega}_{c} L$ on the transverse momentum phase space, see Fig. 3. To estimate the scale $\hat{\omega}$ at which this suppression sets in, we parallel the argument leading to Eq. (2.12). We require that the characteristic angle of the gluon emission is of order one, finding

$$
\Theta_{c}^{2} \simeq \frac{\mu^{2}}{\hat{\omega}^{2}} \simeq\left(\frac{\bar{\omega}_{c}}{\hat{\omega}}\right)^{2} \frac{1}{\bar{R}} \sim 1 \Rightarrow \frac{\hat{\omega}}{\bar{\omega}_{c}} \propto \frac{1}{\sqrt{\bar{R}}} .
$$

The numerical position of the maximum of $\omega\left(d I^{N=1} / d \omega\right)$ in Fig. 3 changes $\propto 1 / \sqrt{\bar{R}}$, in accordance with this estimate. We thus have a semiquantitative understanding of how phase space constraints deplete the nonperturbative soft region of the medium-induced gluon energy distribution.

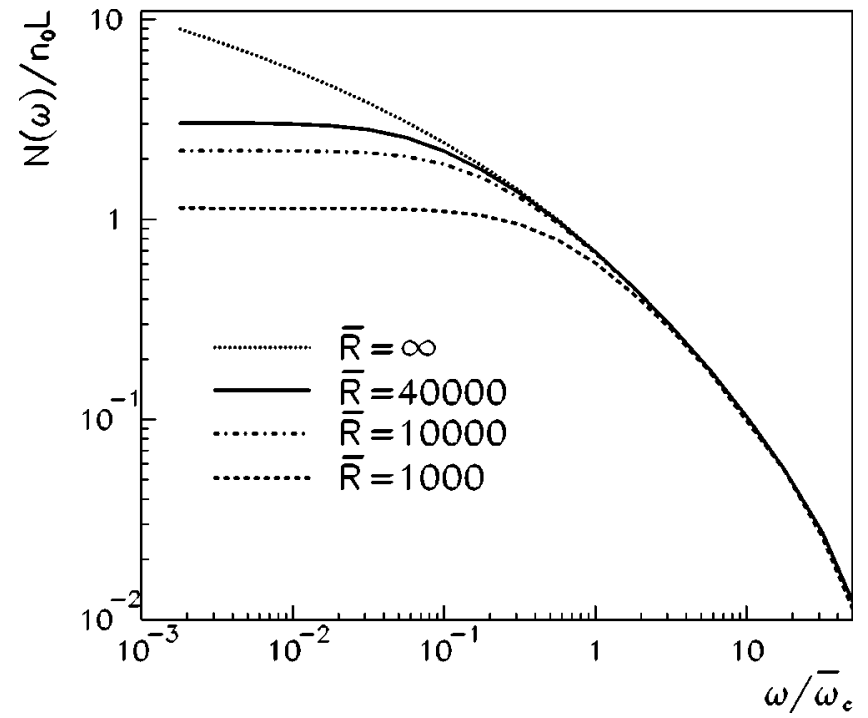

FIG. 4. The multiplicity of additional medium-induced gluons (2.14) radiated with energy larger than $\omega$. Calculation done in the single hard scattering approximation.

In Fig. 4, the additional medium-induced gluon multiplicity (2.14) is calculated in the opacity approximation. In the absence of kinematic constraints $\bar{R}=\infty(R=\infty)$ and for sufficiently small gluon energies $\omega<\bar{\omega}_{c}\left(\omega<\omega_{c}\right)$, this multiplicity changes $\propto 1 / \omega$ in the opacity approximation $(\propto 1 / \sqrt{\omega}$ in the multiple soft scattering approximation). In the presence of kinematic constraints, the total additional multiplicity is comparable for both approximations: $N(\omega=0) \leqslant 3$.

\section{Comparison: multiple soft vs single hard scattering approximation}

Qualitative. The squared transverse momentum accumulated by a projectile due to Brownian motion increases linearly $\propto \mu^{2} n_{0} L$ with path length where $n_{0}=1 / \lambda$ denotes the longitudinal density of scattering centers. This leads to

$$
\mu^{2} n_{0} L=\hat{q} L \text { for Brownian motion }
$$

and thus

$$
\omega_{c}=\frac{1}{2} \hat{q} L^{2}=\frac{L}{\lambda} \bar{\omega}_{c} \quad \text { in the multiple soft scattering limit. }
$$

Recent applications of the opacity approximation use 1 $\leqslant L / \lambda \leqslant 3$. In this case, the gluon energy distribution is much harder in the opacity approximation than in the multiple soft scattering approximation, see Fig. 5.

Quantitative. The relation $\omega_{c}=\left(n_{0} L\right) \bar{\omega}_{c}$ holds only if the projectile accumulates transverse momentum by Brownian motion. In general, deviations from Brownian motion are due to the high transverse momentum tails of the elastic scattering cross sections 


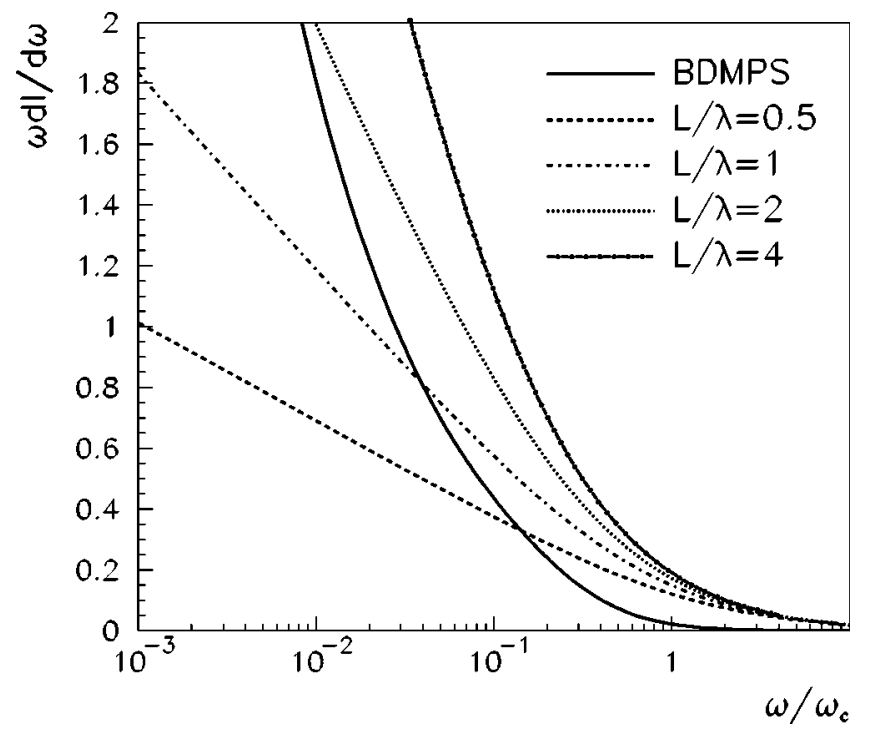

FIG. 5. The gluon energy distribution without kinematic constraint $(R, \bar{R} \rightarrow \infty)$ as calculated in the multiple soft scattering approximation, and in the single hard scattering approximation for $n_{0} L=0.5,1,2,4$. Results for the single hard scattering approximation are plotted for $(L / \lambda) \bar{\omega}_{c}=\omega_{c}$.

$$
|a(\mathbf{q})|^{2}=\frac{\mu^{2}}{\pi\left(\mathbf{q}^{2}+\mu^{2}\right)^{2}} .
$$

In QED, the Coulomb scattering distribution is well represented by the theory of Molière [15] and shows logarithmic deviations from Brownian motion. For QCD, one can identify an analogous logarithmic term in the transport coefficient (2.3) by expanding the dipole cross section (2.2)

$$
\hat{q} L=n_{0} L \int \frac{d^{2} \mathbf{q}}{(2 \pi)^{2}}|a(\mathbf{q})|^{2} \frac{1}{2} \mathbf{q}^{2} \cos ^{2} \varphi \sim\left(n_{0} L\right) \mu^{2} \ln \sqrt{\frac{E_{\mathrm{cut}}}{\mu}} .
$$

Here, $E_{\text {cut }}$ denotes the upper cutoff of the logarithmically divergent $q$ integral. This changes Eq. (2.26) to

$$
\omega_{c}=\left(n_{0} L\right) \bar{\omega}_{c} \ln \sqrt{\frac{E_{\mathrm{cut}}}{\mu}} .
$$

The logarithmic term makes the comparison between single hard and multiple soft scattering approximation more difficult. Based on Eq. (2.29), the curves for the single hard scattering approximation should be shifted in Fig. 5 by a factor $\ln \sqrt{E_{\text {cut }} / \mu}>1$ to the left. For realistic values $\left[\mu \geqslant \Lambda_{\mathrm{QCD}}\right.$ and $E_{\text {cut }} \leqslant E$ say], we find $\ln \sqrt{E_{\text {cut }} / \mu} \ll 10$. Thus, the above conclusion stays unchanged: the medium-induced gluon energy distribution is significantly harder in the single hard scattering approximation than in the multiple soft one.

\section{QUENCHING WEIGHTS}

Medium-induced gluon radiation modifies the correspondence between the initial parton and the final hadron momenta. This modification can be determined from the distri- bution $P(\Delta E)$ of the additional medium-induced energy loss which we calculate now. If gluons are emitted independently, $P(\Delta E)$ is the normalized sum of the emission probabilities for an arbitrary number of $n$ gluons which carry away the total energy $\Delta E$ :

$$
\begin{aligned}
P(\Delta E)= & \sum_{n=0}^{\infty} \frac{1}{n !}\left[\prod_{i=1}^{n} \int d \omega_{i} \frac{d I\left(\omega_{i}\right)}{d \omega}\right] \delta\left(\Delta E-\sum_{i=1}^{n} \omega_{i}\right) \\
& \times \exp \left[-\int_{0}^{\infty} d \omega \frac{d I}{d \omega}\right] .
\end{aligned}
$$

The summation over arbitrarily many gluon emissions in Eq. (3.1) can be performed by Laplace transformation [1]

$$
\begin{aligned}
P(\Delta E) & =\int_{C} \frac{d \nu}{2 \pi i} \mathcal{P}(\nu) e^{\nu \Delta E}, \\
\mathcal{P}(\nu) & =\exp \left[-\int_{0}^{\infty} d \omega \frac{d I(\omega)}{d \omega}\left(1-e^{-\nu \omega}\right)\right] .
\end{aligned}
$$

Here, the contour $C$ runs along the imaginary axis with Re $\nu=0$. In general, the probability distribution $P(\Delta E)$ has a discrete and a continuous part [11]

$$
P(\Delta E)=p_{0} \delta(\Delta E)+p(\Delta E)
$$

The discrete weight $p_{0}$ may be viewed as the probability that no additional gluon is emitted due to in-medium scattering and hence no medium-induced energy loss occurs. This weight is determined by the total gluon multiplicity

$$
p_{0}=\lim _{\nu \rightarrow \infty} \mathcal{P}(\nu)=\exp [-N(\omega=0)]
$$

For finite in-medium path length, there is always a finite probability $p_{0} \neq 0$ that the projectile is not affected by the medium. Only a finite number of additional medium-induced gluons can be emitted, see Eq. (3.5). For infinite in-medium path length, one finds

$$
\lim _{R \rightarrow \infty} p_{0}=0
$$

The medium-induced gluon energy distribution $\omega(d I / d \omega)$ determines to what extent the total energy distribution of a given parton deviates from its "vacuum" fragmentation in an elementary collision

$$
\omega \frac{d I^{(\mathrm{tot})}}{d \omega}=\omega \frac{d I^{(\mathrm{vac})}}{d \omega}+\omega \frac{d I}{d \omega} .
$$

From the Laplace transform (3.2), we obtain for the corresponding total probability

$$
P^{(\mathrm{tot})}(\Delta E)=\int_{0}^{\infty} d \bar{E} P(\Delta E-\bar{E}) P^{(\mathrm{vac})}(\bar{E}) .
$$

The probability $P^{(\text {tot })}(\Delta E)$ is normalized to unity and it is positive definite. In contrast, the medium-induced modifica- 
tion of this probability $P(\Delta E)$ is a generalized probability. It can take negative values for some range in $\Delta E$, as long as its normalization is unity,

$$
\int_{0}^{\infty} d \bar{E} P(\bar{E})=p_{0}+\int_{0}^{\infty} d \bar{E} p(\bar{E})=1 .
$$

In this section, we calculate $P(\Delta E)$ in the multiple soft and single hard scattering approximations. The results of these quenching weights are available as a FORTRAN routine [16].

\section{A. Quenching weights in the multiple soft scattering approximation}

By numerical evaluation of the Laplace transform (3.2), (3.3), we have calculated quenching weights $P(\Delta E)$ for the medium-induced energy distribution (2.1) in the multiple soft scattering approximation. To motivate the range of parameter values studied in what follows, we relate the transport coefficient $\hat{q}$ to the in-medium path length $L$ and the saturation scale $Q_{s}[10]$,

$$
Q_{s}^{2} \simeq \hat{q} L .
$$

The saturation momentum $Q_{s}$ determines the total average transverse momentum transferred to the partonic projectile in the high-energy limit of totally coherent scattering. Phenomenological estimates for $Q_{s}$ are very uncertain but $Q_{s}^{2} \leqslant(3 \mathrm{GeV})^{2}$ may be considered as an upper bound at CERN Large Hadron Collider (LHC). This is also consistent with alternative estimates $[2,6,10]$ for the transport coefficient $\hat{q}$. To discuss in-medium path lengths $L$ up to twice a nuclear $\mathrm{Pb}$ radius, we thus have to explore the parameter space up to $R<40000$. We choose a very small lower value $R=\chi^{2} \omega_{c} L^{3}=1$ in order to tabulate quenching weights for the radiation outside very small opening angles $\chi=\sin \Theta$. All results will be given for energies in units of $\omega_{c}$.

The discrete weight $p_{0}$ of the probability distribution $P(\Delta E)$ is plotted in Fig. 6 as a function of the kinematic cutoff $R=\omega_{c} L$. It approaches unity in the absence of a medium $(R \rightarrow 0)$ and it vanishes in the limit of infinite inmedium path length, see Eq. (3.6). Remarkably, $p_{0}$ exceeds unity for small values $R<100$. This indicates that there is a phase space region at very small transverse momentum, into which less gluons are emitted in the medium than in the vacuum. The "vacuum" gluon radiation is shifted to larger transverse momentum in the presence of a medium [6]. The decrease of the discrete weight for large $R$ and its growth above unity for sufficiently small $R$ both depend on the strength of the interaction between partonic projectile and medium. They are thus more pronounced for gluons than for quarks.

The continuous part $p(\Delta E)$ of the probability distribution (3.4) is shown in Fig. 7 as a function of the dimensionless energy fraction $\Delta E / \omega_{c}$ for different values of the kinematic constraint $R$. Increasing the density of the medium (i.e., increasing the transport coefficient $\hat{q}$ ) or increasing the inmedium path length $L$ corresponds to an increase of $\omega_{c}$ and $R$. Figures 6 and 7 specify how the probability that the parton

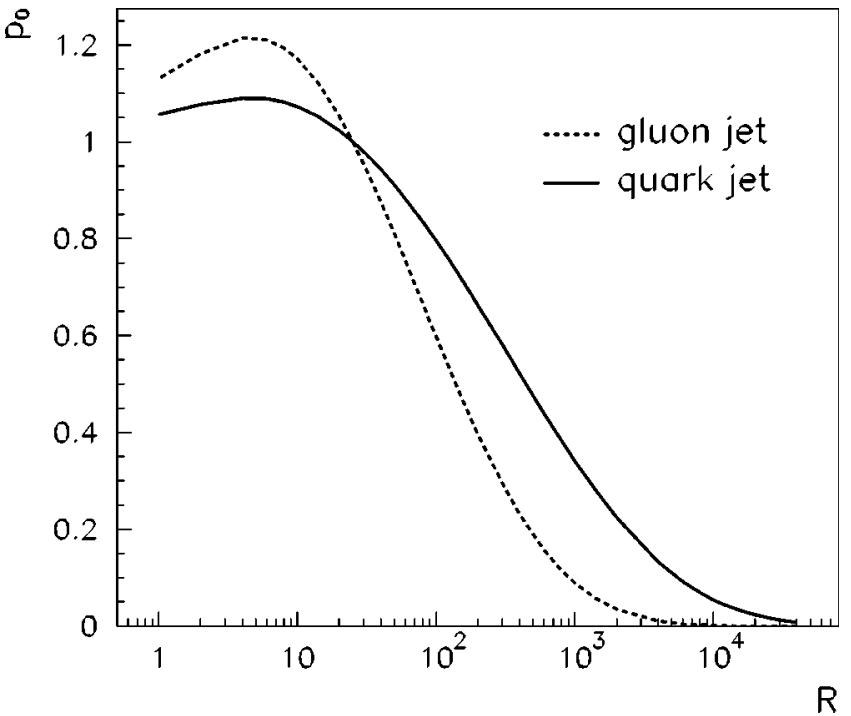

FIG. 6. The discrete part $p_{0}$ of the quenching weight (3.4) calculated in the multiple soft scattering approximation as a function of $R=\omega_{c} L$.

loses an energy fraction $\Delta E$ changes with these medium properties. As expected from the normalization (3.9), the continuous part $p(\Delta E)$ shows predominantly negative contributions for small values $R<100$ where the discrete weight $p_{0}$ exceeds unity.

In the limit $R \rightarrow \infty$, the quenching weight was found to be fit very well by a two-parameter log-normal distribution [17]. This is a heuristic observation which is difficult to connect to the analytical structure of the gluon energy distribution. Analytically, an estimate of the quenching weight can be obtained [1] in the limit $R \rightarrow \infty$ from the small- $\omega$ approximation $\omega(d I / d \omega) \propto 1 / \sqrt{\omega}$ in Eq. (2.10),
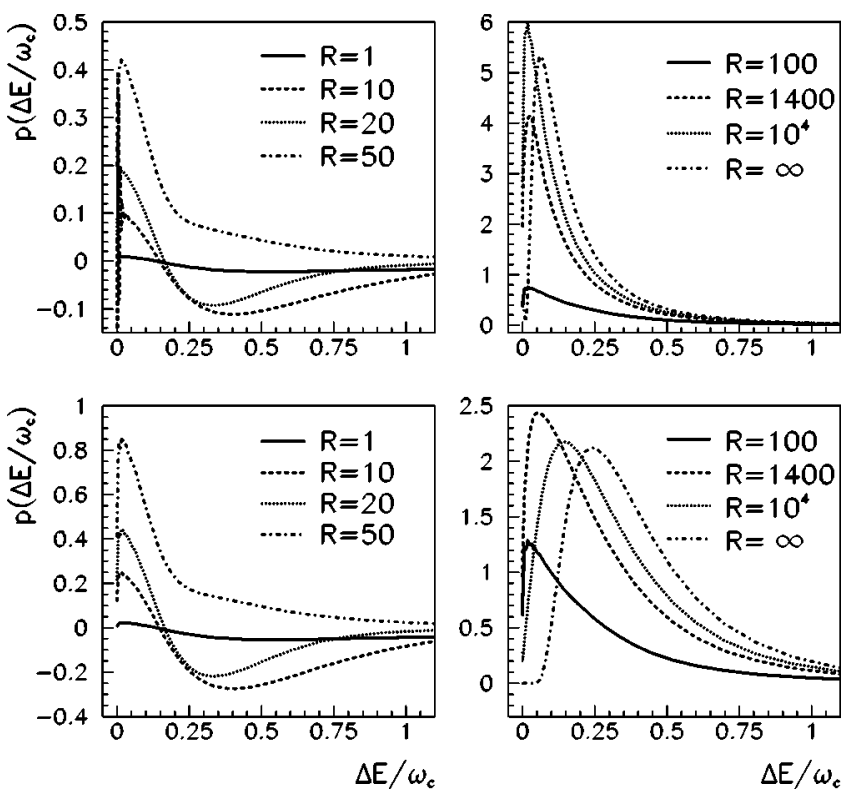

FIG. 7. The continuous part of the quenching weight (3.4), calculated in the multiple soft scattering approximation for a hard quark (upper row) or hard gluon (lower row). 


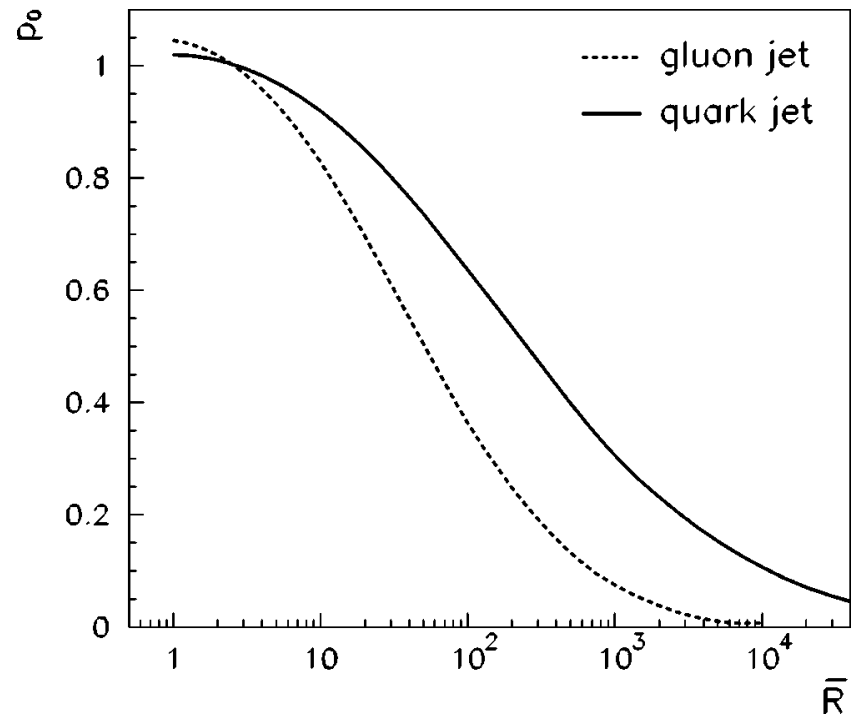

FIG. 8. The discrete part $p_{0}$ of the quenching weight calculated in the single hard scattering approximation for opacity $n_{0} L=1$.

$$
P_{\mathrm{BDMS}}^{\mathrm{approx}}(\epsilon)=\sqrt{\frac{a}{\epsilon^{3}}} \exp \left[-\frac{\pi a}{\epsilon}\right], \text { where } a=\frac{2 \alpha_{s}^{2} C_{R}^{2}}{\pi^{2}} \omega_{c} \text {. }
$$

This approximation is known to capture [11] the rough shape of the probability distribution for large system size, but it has an unphysical large $\epsilon$ tail with infinite first moment $\int d \epsilon \epsilon P_{\mathrm{BDMS}}^{\text {approx }}(\epsilon)$. Also, its maximum $\epsilon_{\max }=2 a \pi / 3$ grows stronger with the effective coupling $\alpha_{s} C_{R}$ than the numerical result in Fig. 7.

\section{B. Quenching weights in the opacity approximation}

We have evaluated the quenching weight (3.4) for the medium-induced gluon energy distribution in the $N=1$ opacity approximation (2.19). In general, the quenching weight depends in this approximation on the characteristic gluon energy $\bar{\omega}_{c}$, the kinematic constraint $\bar{R}=\chi^{2} \bar{\omega}_{c} L$, and the opacity $n_{0} L$.

For the numerical results presented in Figs. 8 and 9, we use $n_{0} L=1$. The gluon energy distribution $\omega(d I / d \omega)$ depends linearly on $n_{0} L$, but the quenching weight shows a complicated dependence on $n_{0} L$; it has to be calculated separately for each value of $n_{0} L$ from Eqs. (3.2) and (3.3). However, since $n_{0} L$ multiplies the Casimir factor $C_{R}$ in the gluon energy distribution, the quenching weight for gluons with $n_{0} L=1$ is identical to the quenching weight for quarks with $n_{0} L=C_{A} / C_{F}=2.25$. Vice versa, the quenching weight for quarks given in Figs. 8 and 9 can be viewed as a quenching weight for gluons with $n_{0} L=C_{F} / C_{A}$.

\section{Comparison: multiple soft vs single hard scattering approximation}

In the opacity approximation, one specifies both the average transverse momentum squared $\sim \mu^{2} n_{0} L$ transferred to the projectile and the average number $n_{0} L$ of scattering cen-
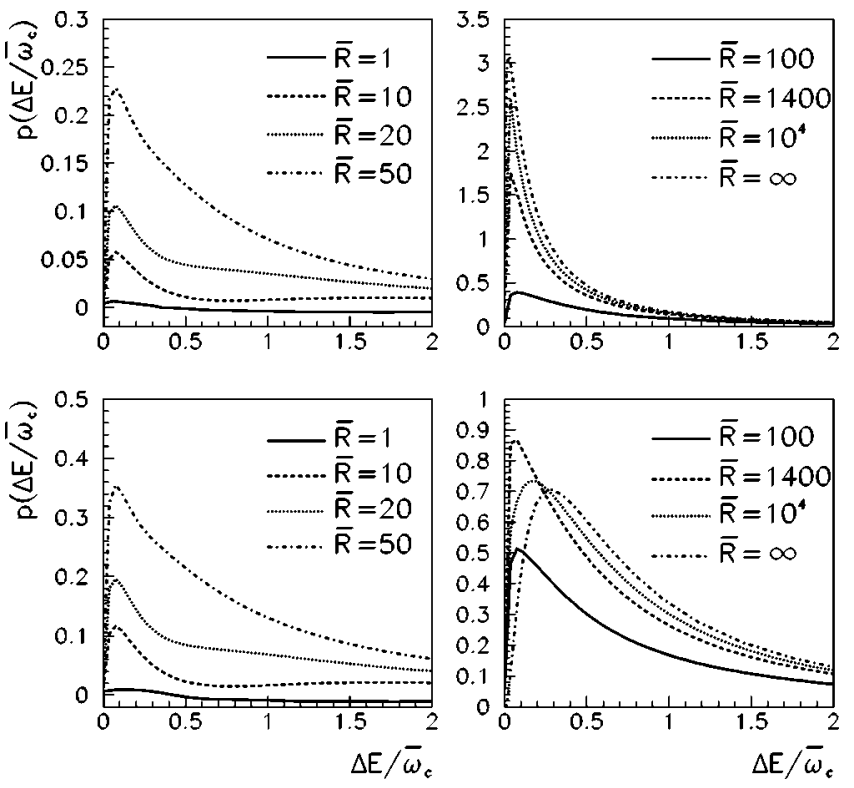

FIG. 9. The continuous part of the quenching weight calculated in the single hard scattering approximation with opacity $n_{0} L=1$ for a hard quark (upper row) or hard gluon (lower row).

ters involved in this momentum transfer. This is in contrast to the multiple soft scattering approximation which specifies the average transverse momentum squared transferred to the projectile irrespective of the number of scattering centers involved. Thus, the single hard scattering approximation contains one additional model parameter, the opacity $n_{0} L$.

Despite this difference, we want to compare the quenching weights obtained in both approximations. To this end, we start from the relations

$$
R \simeq\left(n_{0} L\right) \bar{R}, \omega_{c} \simeq\left(n_{0} L\right) \bar{\omega}_{c},
$$

discussed in Sec. II C. Keeping the values of $R, \omega_{c}$ and $\bar{R}, \bar{\omega}_{c}$ fixed, we "fit" the opacity $n_{0} L$ such that the quenching weights obtained in both approximations show the best agreement. This allows us to discuss for both approximations differences in functional shape which cannot be removed by a change of model parameters.

We start by comparing the $R$ and $\bar{R}$ dependences of the discrete weight $p_{0}$ calculated in the multiple soft (Fig. 6) and single hard (Fig. 8) scattering approximation, respectively. For the choice $R \simeq 3 \bar{R}$, the curves show better agreement. However, the excess above unity for $R<100$ is much more pronounced in the multiple soft scattering approximation, than the excess above unity for $3 \bar{R}<100$ in the single hard scattering approximation. This indicates that the specific destructive interference effects discussed in Sec. III A play a more important role in the multiple soft scattering approximation.

In Fig. 10, we compare both approximations in the limit in which the constraint on the transverse momentum phase space is removed (i.e., $R, \bar{R} \rightarrow \infty$ ). For the opacity $n_{0} L=3$, the maximum of the quenching weight takes the same value in both approximations. However, significant differences can 


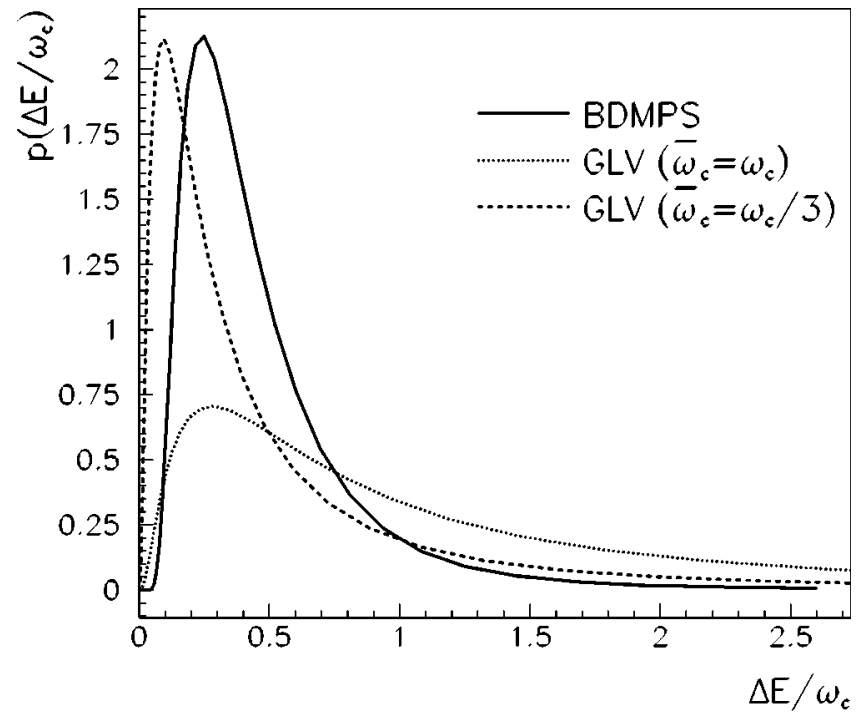

FIG. 10. Comparison of the quenching weights for infinite system size $(R, \bar{R} \rightarrow \infty)$ calculated for a hard gluon in the multiple soft ("BDMPS") and single hard ("GLV") scattering approximation. For rescaled characteristic gluon energy, $\omega_{c} \simeq\left(n_{0} L\right) \bar{\omega}_{c}, n_{0} L=3$, the agreement between both probability distributions improves, see text for further discussion.

be seen in the functional shape. The gluon energy distribution is harder in the single hard scattering approximation (see Fig. 5) and this is reflected in a more pronounced large energy tail of the quenching weight. We regard the remaining differences between both approximations as an indication of the intrinsic theoretical uncertainties in evaluating the gluon energy distribution (2.1).

\section{MEDIUM-INDUCED GLUON RADIATION FOR AN EXPANDING MEDIUM}

Hard partons produced in the initial stage of ultra relativistic nucleus-nucleus collisions are propagating through a strongly expanding medium. This results in a time dependence of the transport coefficient $\hat{q}(\xi)$ which can be parametrized in terms of a power law

$$
\hat{q}(\xi)=\hat{q}_{0}\left(\frac{\xi_{0}}{\xi}\right)^{\alpha}
$$

The expansion parameter $\alpha$ determines the dynamical evolution of the medium: $\alpha=0$ characterizes a static medium. A one-dimensional, boost-invariant longitudinal expansion is described by $\alpha=1$. This value is supported by hydrodynamical simulations of the early stage. In general, however, an additional transverse expansion can lead to larger values $\alpha$ $\leqslant 3$. The maximal value $\hat{q}_{0}$ of the transport coefficient is reached at the time of highest density of the system which is the formation time $\xi_{0}$. This formation time may be set by the inverse of the saturation scale $p_{\text {sat }}[18]$, resulting in $\approx 0.2 \mathrm{fm} / c$ at RHIC and $\approx 0.1 \mathrm{fm} / c$ at LHC. The difference between $\xi_{0}$ and the production time of the hard parton is negligible for the calculation of the gluon energy distribution

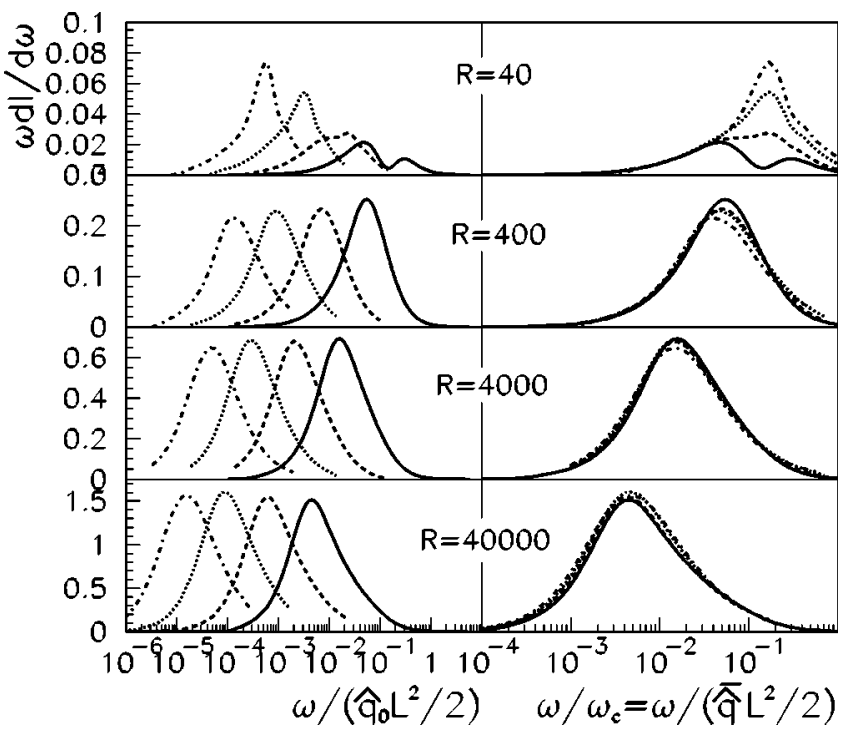

FIG. 11. The gluon energy distribution calculated in the multiple soft scattering approximation with expansion parameter $\alpha=0$ (solid line), $\alpha=0.5$ (dashed line), $\alpha=1.0$ (dotted line), and $\alpha=1.5$ (dashdotted line). Curves in the right hand column are scaled according to Eq. (4.2).

(2.1). It will be ignored in what follows. In this section, we discuss the range of validity and the form of a dynamical scaling law [11] which relates the gluon energy distribution (2.1) in a collision of arbitrary dynamical expansion to an equivalent static scenario.

\section{A. Multiple soft scattering in an expanding medium}

In Appendix $\mathrm{C}$, we give details of the calculation of the gluon energy distribution (2.1) for values of the expansion parameter $\alpha<3$ in the multiple soft scattering approximation (2.3). As reported previously [11], we observe a scaling law which relates the time-dependent transport coefficient (4.1) to an equivalent static transport coefficient $\overline{\hat{q}}$,

$$
\bar{q}=\frac{2}{L^{2}} \int_{\xi_{0}}^{L+\xi_{0}} d \xi\left(\xi-\xi_{0}\right) \hat{q}(\xi)
$$

As seen in Fig. 11, the gluon energy distributions for different values of the expansion parameter $\alpha$ differ by orders of magnitude if plotted in units of the same characteristic gluon energy $\omega_{c}=\frac{1}{2} \hat{q}_{0} L^{2}$ and kinematic constraint $R=\omega_{c} L$. However, if plotted in units of the rescaled gluon energy $\frac{1}{2} \overline{\hat{q}} L^{2}$ and the rescaled kinematic constraint $\frac{1}{2} \overline{\hat{q}} L^{3}$, they agree approximately over a large parameter range.

For practical purposes, the accuracy of the scaling law (4.2) is satisfactory for $R>100$. Concerning the deviations from the scaling law for $R<100$ (see Fig. 11), we make the following comments: In practice, these deviations are negligible since $p_{0} \sim 1$ for $R<100$ and thus no significant medium modification occurs. Technically, the static case ( $\alpha$ $=0$ ) is calculated for a box profile in the longitudinal density of scattering centers. On the other hand, in the expanding 
scenarios, the density profile degrades more smoothly with increasing path length, and the discontinuity at path length $L$ is less important. The strength of destructive interferences between medium-induced and vacuum gluon radiation depends on this discontinuity. This may explain why for $R$ $=40$ the rescaled gluon energy distribution in Fig. 11 is more suppressed in the static case than in the expanding case.

\section{B. The opacity expansion for an expanding medium}

In Appendix B, we give analytical expression for the single hard scattering limit of the gluon energy distribution (2.1) in a medium with expansion parameter $\alpha$. The analytical form of Eq. (2.1) changes with the expansion parameter $\alpha$. We derive an explicit expression for the Bjorken scaling case $\alpha=1$

$$
\begin{aligned}
\omega \frac{d I_{\alpha=1}^{N=1}}{d \omega}= & 2 \frac{\alpha_{s} C_{R}}{\pi}\left(n_{0} \xi_{0}\right) \\
& \times \int_{0}^{\infty} \frac{d r}{r} \operatorname{Re}\left[-\mathrm{Ei}[-i r]+\ln [-i r]+\gamma_{E}\right] \\
& \times\left(\frac{\gamma}{r+\gamma}-\frac{\gamma}{\sqrt{\left(\kappa^{2}+r+\gamma\right)^{2}-4 \kappa^{2} r}}\right)
\end{aligned}
$$

Here, $\gamma_{E} \approx 0.577 \cdots$ denotes Euler's constant and the exponential integral function is $\mathrm{Ei}[z]=-\int_{-z}^{\infty} d t e^{-t} / t$.

To relate the gluon energy distributions for a static medium (2.19) and a Bjorken scaling expansion (4.3), we determine the dynamically averaged density of scattering centers following Eq. (4.2)

$$
\bar{n}=\frac{2}{L^{2}} \int_{\xi_{0}}^{L+\xi_{0}} d \xi\left(\xi-\xi_{0}\right) n(\xi)=\frac{2 n_{0} \xi_{0}}{L} .
$$

This equation suggests that the gluon energy distributions in the static and Bjorken expansion case show agreement if the prefactor $\left(n_{0} \xi_{0}\right)$ in Eq. (4.3) is replaced by $\frac{1}{2} \bar{n} L$ where $\bar{n}$ determines the density of scattering centers of the equivalent static scenario. In Fig. 12, we test this suggestion numerically for different values of the kinematic constraint $\bar{R}$.

Remarkably, for sufficiently large kinematic constraint $\bar{R}$ $>100$, the gluon energy distribution shows dynamical scaling according to Eq. (4.4) for gluon energies $\omega>\bar{\omega}_{c}$. This is the dominant kinematic region in the opacity expansion [see the discussion in Sec. II B, Eq. (2.21) ff]. Thus, despite the deviations from the scaling law for $\omega<\bar{\omega}_{c}$, the logarithmically enhanced contribution to the average energy loss

$$
\langle\Delta E\rangle^{N=1}=\int d \omega \omega \frac{d I^{N=1}}{d \omega}
$$

shows scaling [19] with Eq. (4.4). This is also known to hold in the soft multiple scattering approximation [20] and it is consistent with results obtained on the basis of twist-4 matrix elements [21].
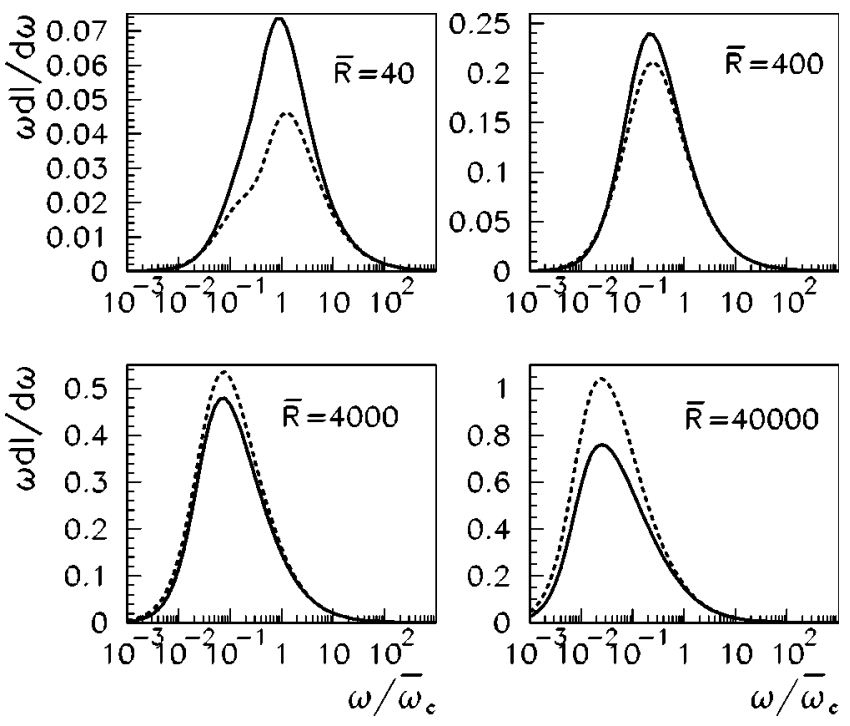

FIG. 12. The gluon energy distribution calculated in the single hard scattering approximation for a static medium (dashed line) and for a medium with Bjorken expansion (solid line). Curves for the dynamically expanding case are scaled according to Eq. (4.4).

In the region $\omega<\bar{\omega}_{c}$, significant deviations from the scaling law (4.4) are seen in Fig. 12. However, the logarithmic plot overemphasizes the importance of these deviations. First, they occur in the sub dominant region which is less important for calculating the quenching weights. Second, these deviations do not exceed $30 \%$ in the physically relevant parameter range $100<\bar{R}<40000$ in which significant medium modifications can be expected.

For practical purposes, the scaling law (4.4) is thus satisfactory. Quenching weights for a dynamically expanding scenario can be obtained by calculating the quenching weights of the dynamically equivalent static scenario according to Eq. (4.4).

\section{ANGULAR DEPENDENCE OF RADIATION PROBABILITY}

The maximal angle under which a gluon can be radiated is given by the upper bound on the transverse momentum integral in Eq. (2.1),

$$
\Theta \simeq \frac{k_{\perp}^{\max }}{\omega}=\chi
$$

Thus, for fixed values of the characteristic gluon energy $\omega_{c}$ and of the kinematic constraint $R=\omega_{c} L$, a decreasing value of $R_{\chi}=\chi^{2} \omega_{c} L$ determines the medium-induced energy radiated into a cone of opening angle $\Theta$. In this section, we denote explicitly the dependence of the quenching weight $P\left(\Delta E, \omega_{c}, R_{\chi}\right)$ on $\omega_{c}$ and $\chi^{2} R$. This quenching weight determines the probability that an additional energy fraction $\Delta E$ is radiated inside the opening angle $\chi=\Theta$. From the Figs. 1 and 3, we know that the more collinear component of the medium-induced gluon radiation is harder. 

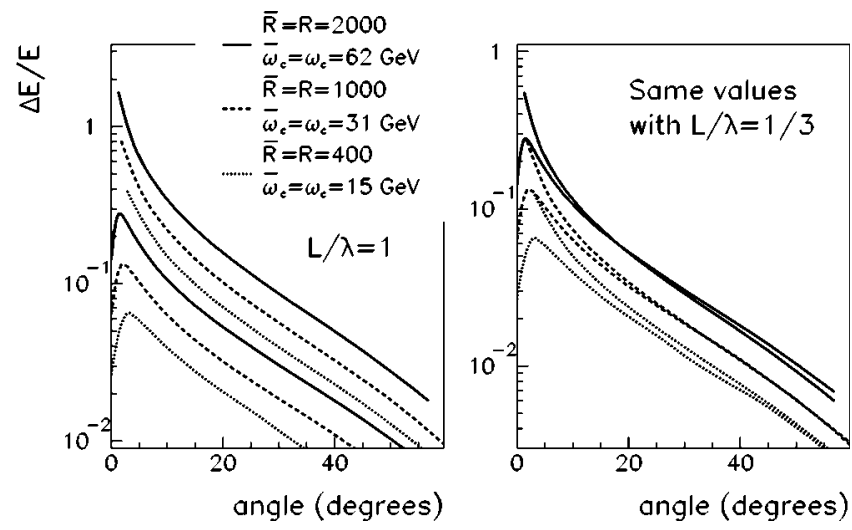

FIG. 13. The average energy loss (5.3) radiated outside an angle $\Theta$ as calculated in the multiple soft (lower three lines) and single hard (upper three lines) scattering approximation for a jet of energy $E=100 \mathrm{GeV}$.

For fixed values of $\omega_{c}$ and $R=\omega_{c} L$, the gluon energy distribution radiated outside the opening angle $\Theta$ is given by

$$
\omega \frac{d I^{>\Theta}}{d \omega}\left(\omega_{c}, R\right)=\omega \frac{d I}{d \omega}\left(\omega_{c}, R\right)-\omega \frac{d I}{d \omega}\left(\omega_{c}, R_{\chi}\right) .
$$

The probability that an additional energy fraction $\Delta E$ is radiated outside the opening angle $\chi \simeq \Theta$ is obtained by inserting Eq. (5.2) into the Mellin transform (3.2), (3.3). For the current work, we did not calculate this probability; there is no simple way to obtain it without Mellin transform directly from the quenching weights tabulated in Sec. III.

The calculation of the average energy loss outside an angle $\Theta$ is simpler. It can be calculated from the quenching weights tabulated in Sec. III

$$
\begin{aligned}
\langle\Delta E\rangle(\Theta)= & \int d \omega \omega \frac{d I^{>\Theta}}{d \omega}\left(\omega_{c}, R=\omega_{c} L\right) \\
= & \int d \bar{E} \bar{E}\left[P\left(\bar{E}, \omega_{c}, R=\omega_{c} L\right)\right. \\
& \left.-P\left(\bar{E}, \omega_{c}, R_{\chi}=\chi^{2} \omega_{c} L\right)\right] .
\end{aligned}
$$

In Fig. 13, we compare the angular dependence of the average parton energy loss (5.3) in the multiple soft and single hard scattering approximation.

In the single hard scattering approximation, the integral (5.3) diverges logarithmically in the ultraviolet for $\chi=\Theta$ $\rightarrow 0$. For the calculation of $\langle\Delta E\rangle^{N=1}(\Theta=0)$ in Eq. (2.22), we have cut off this divergence by limiting the energy radiated away to $\omega<E$. For the plot in Fig. 13, we restrict instead the calculation to sufficiently large angles $\Theta$ for which the second term in Eq. (5.3) provides an ultraviolet cutoff. Thus, for small angles $\Theta<10^{\circ}$ where $\Delta E \sim E$, one overestimates $\langle\Delta E\rangle^{N=1}(\Theta)$.

In the single hard scattering approximation, the region $\omega$ $>\bar{\omega}_{c}$ is dominant. This hard part of the spectrum is emitted under angles $\Theta<\mu / \bar{\omega}_{c}$ and thus appears as a logarithmic enhancement in the collinear region. In the multiple soft scattering approximation, however, the dominant radiative contribution lies in the soft region $\omega<\omega_{c}$ which is emitted under relatively large opening angles $\Theta>k_{\perp} / \omega_{c}$ $\sim \hat{q}^{1 / 4} / \omega_{c}^{3 / 4}$. For smaller opening angles, the average energy loss $\langle\Delta E\rangle(\Theta)$ does not increase further. Indeed, multiple soft scattering results in a shift in transverse phase space which is known to deplete $\langle\Delta E\rangle(\Theta)$ at very small angles $[6,22]$. Thus, in the multiple soft scattering approximation, there is no ultraviolet divergence at small angle $\Theta$.

To compare the single hard and multiple soft scattering approximations for $\langle\Delta E\rangle(\Theta)$, we proceed in analogy to the discussion in Sec. III C: varying the opacity, we find the best agreement between both approximations for $n_{0} L=3$. A qualitative difference which cannot be adjusted by the choice of the additional model parameter $n_{0} L$ persists for small angles only. Its origin is explained above. Thus, Fig. 13 indicates that for comparable sets of model parameters $\omega_{c}, R$ and $\bar{\omega}_{c}, \bar{R}, n_{0} L$, respectively, the multiple soft and single hard scattering approximations lead to a comparable angular dependence of $\langle\Delta E\rangle(\Theta)$ for $\Theta>10^{\circ}$.

The calculation of the angular dependence in Fig. 13 was done for quenching weights calculated for a static medium. In general, the dynamical scaling laws (4.2) and (4.4) relate these to the quenching weights of dynamically expanding scenarios. However, this is not the case for the small values $R, \bar{R}<100$ which enter the calculation of the small angular dependence $\left(\Theta<10^{\circ}\right)$ in Eq. (5.3). For these small values of $R, \bar{R}$, dynamical scaling breaks down (see Figs. 11 and 12). Since typical jet cone openings correspond to larger angles $\Theta>10^{\circ}$, we did not make an effort to quantify the remaining dependence of $\langle\Delta E\rangle(\Theta)$ on the collective expansion of the collision region.

In the multiple soft scattering approximation, $\langle\Delta E\rangle(\Theta$ $=0)$ is finite. Baier et al. observed [23] that the ratio $\langle\Delta E\rangle$ $\times(\Theta) /\langle\Delta E\rangle(\Theta=0)$ is a universal quantity which depends solely on $\hat{q} L^{3} \Theta$. Figure 14 shows that this statement remains approximately true in the presence of a finite kinematic constraint $R$.

\section{APPLICATIONS OF QUENCHING WEIGHTS}

In this section, we use quenching weights to calculate in two alternative ways the medium-induced suppression of hadronic high transverse momentum spectra. In Sec. VI A, we determine the quenching factor $Q\left(p_{\perp}\right)$ and in Sec. VI B we calculate medium-modified parton fragmentation functions. In Sec. VI C we finally discuss the relation of both calculations to the nuclear modification factor measured at RHIC.

\section{A. Quenching factors for hadronic spectra}

The medium-dependence of inclusive transverse momentum spectra can be characterized in terms of the quenching factor [1] 


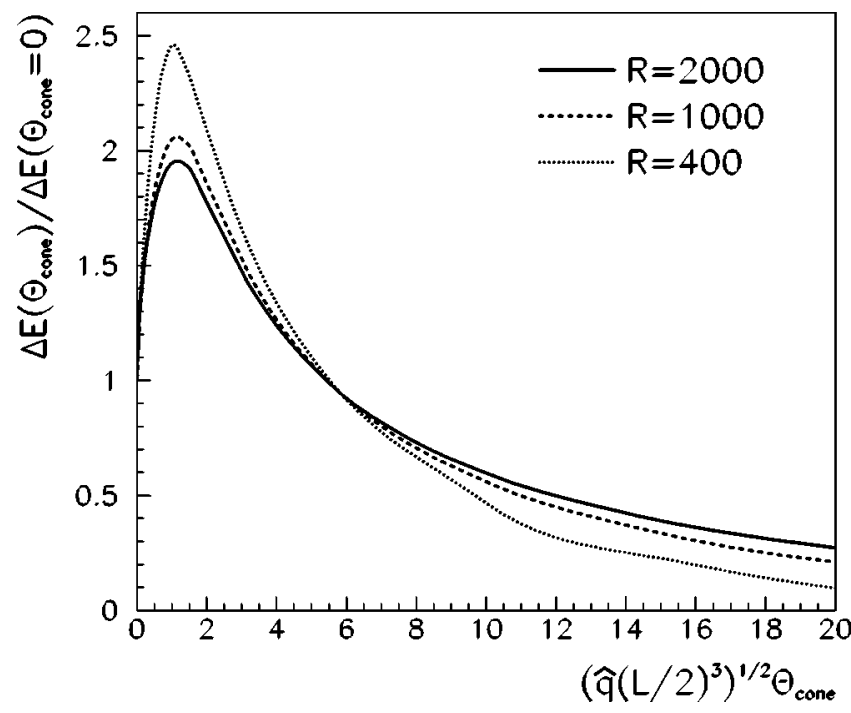

FIG. 14. Angular dependence of the average energy loss (5.3) in the multiple soft scattering approximation as a function of the rescaled parameter $\hat{q}(L / 2)^{3} \Theta$.

$$
\begin{aligned}
Q\left(p_{\perp}\right) & =\frac{d \sigma \operatorname{med}\left(p_{\perp}\right) / d p_{\perp}^{2}}{d \sigma^{\operatorname{vac}\left(p_{\perp}\right) / d p_{\perp}^{2}}} \\
& =\int d \Delta E P(\Delta E)\left(\frac{d \sigma^{\operatorname{vac}}\left(p_{\perp}+\Delta E\right) / d p_{\perp}^{2}}{d \sigma^{\operatorname{vac}}\left(p_{\perp}\right) / d p_{\perp}^{2}}\right) .
\end{aligned}
$$

Here, the spectrum $d \sigma^{\mathrm{vac}}\left(p_{\perp}\right) / d p_{\perp}^{2}$ is unaffected by medium effects; it is determined, e.g., in proton-proton collisions. Equation (6.1) relates it to the medium-modified transverse momentum spectrum $d \sigma^{\text {med }}\left(p_{\perp}\right) / d p_{\perp}^{2}$ measured, e.g., in nucleus-nucleus collisions. We work in the longitudinally comoving frame in which the total energy of the produced parton is directed orthogonal to the beam. Due to the presence of the medium, a parton produced initially with transverse momentum $p_{\perp}+\Delta E$ loses an additional energy $\Delta E$ with probability $P(\Delta E)$. This defines the quenching factor (6.1).

If one assumes a power law fall-off of the $p_{\perp}$ spectrum, then the quenching factor (6.1) can be calculated explicitly,

$$
Q\left(p_{\perp}\right) \simeq \int d \Delta E P(\Delta E)\left(\frac{p_{\perp}}{p_{\perp}+\Delta E}\right)^{n} .
$$

In general, the effective power $n$ depends on $p_{\perp}$ and $\sqrt{s}$. It is $n \simeq 7$ in the kinematic range relevant for RHIC.

To compare directly to published results [1], we calculate the quenching factor (6.2) in Fig. 15 for parameter values used previously. The transport coefficient is taken to match expectations for a hot medium $\hat{q}=(1 \mathrm{GeV})^{2} / \mathrm{fm}$. Given the in-medium path length $L$, this defines $\omega_{c}$ and $R$ in the multiple soft scattering approximation. In the single hard scattering approximation, parameters are chosen for opacity $n_{0} L$ $=1$ by identifying $\bar{R}=R$ and $\bar{\omega}_{c}=\omega_{c}$. The effective power $n$ in Eq. (6.2) is set to its asymptotic value $n=4$. To be quan-

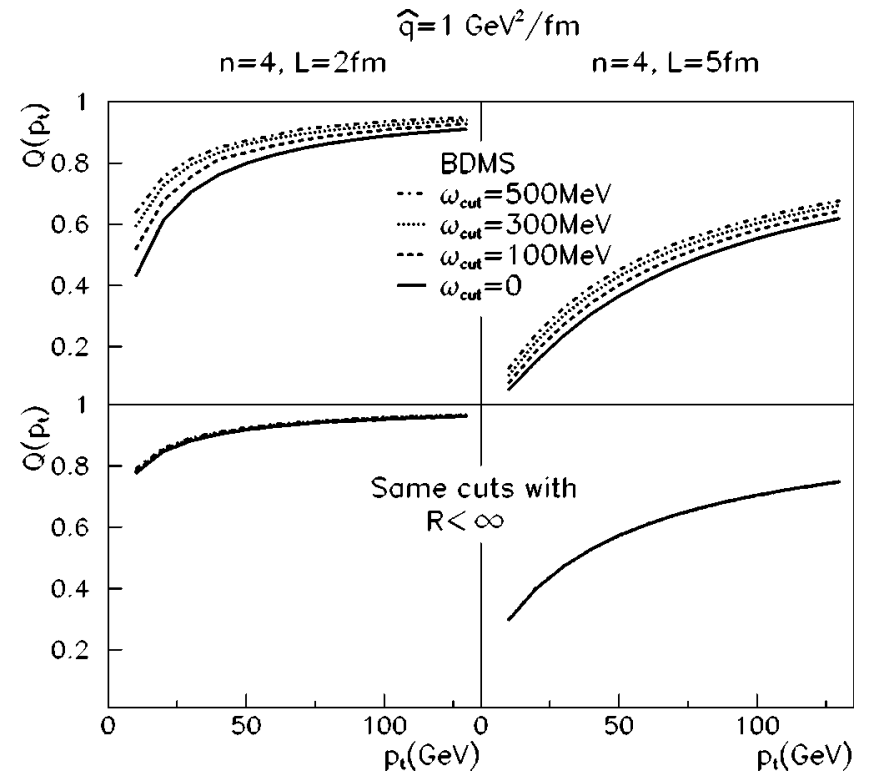

FIG. 15. The quenching factor (6.2) calculated in the soft multiple scattering approximation for $\alpha_{s}=1 / 2$. Upper row: calculation in the $R \rightarrow \infty$ limit but with a varying sharp cutoff on the infrared part of the gluon energy distribution $\omega(d I / d \omega)$ determining the quenching weight. Lower row: the same calculation is insensitive to infrared contributions if the finite kinematic constraint $R=\omega_{c} L$ $<\infty$ is included.

titatively comparable with Ref. [1], we use $\alpha_{s}=1 / 2$ in Figs. 15 and 16 while all other numerical results are given for $\alpha_{s}=1 / 3$.

Figure 15 shows the numerical results obtained in the multiple soft scattering approximation. The perturbative calculation of the gluon energy distribution $\omega(d I / d \omega)$ cannot be trusted for soft gluon energies $\omega \sim O\left(\Lambda_{\mathrm{QCD}}\right)$ where perturbation theory breaks down. To quantify the sensitivity of their calculation to this infrared region, Baier et al. [1] intro-

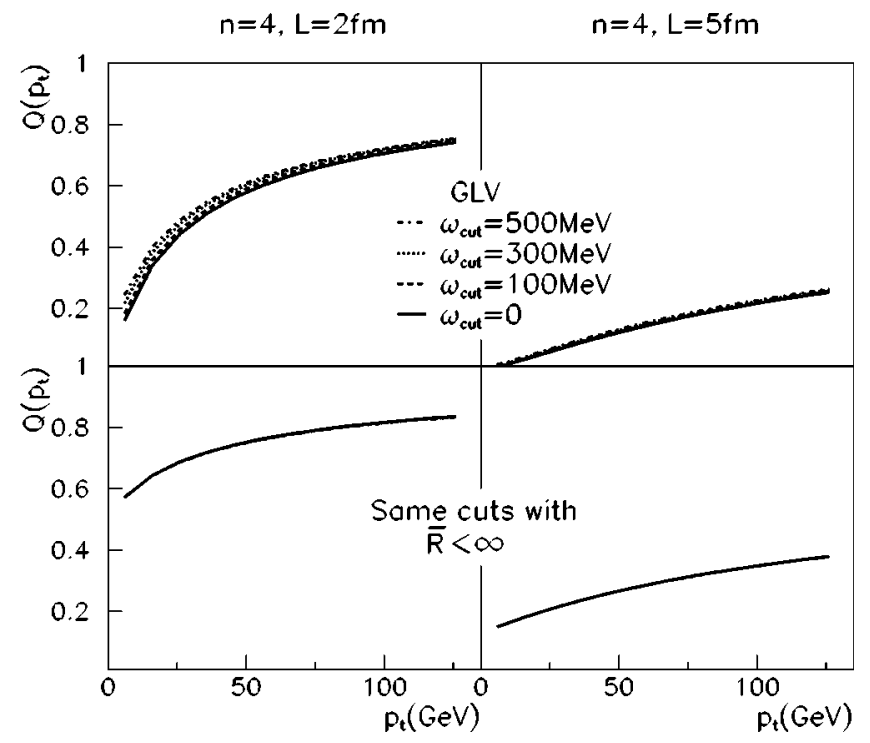

FIG. 16. The same quenching factor (6.2) as in Fig. 15, here calculated in the single hard scattering approximation. 
duced a sharp cutoff on the $R \rightarrow \infty$ gluon energy distribution which was varied between $\omega_{\text {cut }}=0$ and $\omega_{\text {cut }}=500 \mathrm{MeV}$. The resulting uncertainty is seen as a $\approx 20 \%$ variation of the quenching factor $Q\left(p_{\perp}\right)$ in the upper row of Fig. 15. However, the finite kinematic constraint $R=\omega_{c} L<\infty$ depletes the infrared region of the medium-induced gluon radiation spectrum (see Fig. 1). This constraint, which is a generic consequence of a finite in-medium path length, removes almost completely the sensitivity of the calculation to the uncontrolled infrared region (see Fig. 15, lower row). Remarkably, it also tends to flatten the $p_{\perp}$ dependence of the quenching factor. We shall return to the consequences of this observation when we zoom into the region $p_{\perp}<10 \mathrm{GeV}$ in Sec. VI C.

We have calculated the quenching factor (6.1) in the single hard scattering approximation, see Fig. 16. Since the dominant contribution comes in this case from the hard part of the spectrum $\omega>\bar{\omega}_{c}$, the sensitivity to the infrared cut $\omega_{\text {cut }}$ is much reduced in comparison to Fig. 15. However, realistic finite kinematic constraints $\bar{R}$ remove a much larger part of the soft spectrum. As a consequence, the absolute value of $Q\left(p_{\perp}\right)$ increases significantly if finite kinematic constraints are imposed, and the $p_{\perp}$ dependence tends to flatten.

In Sec. III C, we observed that for the quenching weights the best agreement between single hard and multiple soft scattering approximation is for $\omega_{c}=3 \bar{\omega}_{c}, R=3 \bar{R}, n_{0} L=3$. Remaining differences come from the fact that the single hard scattering approximation shows a dominant contribution for $\omega>\omega_{c}$ while the multiple soft scattering approximation shows a dominant contribution for $\omega<\omega_{c}$. However, the quenching factor $Q\left(p_{\perp}\right)$ for small $p_{\perp}$ is sensitive only to the soft region $\omega<\omega_{c},\left(\omega<\bar{\omega}_{c}\right)$ in both approximations. This is so because medium-induced gluons cannot carry away more than the total energy $E_{q}$ of the parent parton, and hence $\omega<E_{q}<\omega_{c}$ at small $p_{\perp}$. Thus, the simple relation $\omega_{c}=3 \bar{\omega}_{c}, R=3 \bar{R}$ does not hold for $p_{\perp}<\omega_{c}$. This is seen in Fig. 17. The dominant multiple soft scattering approximation results in a much stronger suppression than the single hard one calculated for rescaled parameters $\omega_{c}=3 \bar{\omega}_{c}, R=3 \bar{R}$.

\section{B. Medium-modified fragmentation functions}

Medium-induced gluon radiation off hard partons modifies the fragmentation and hadronization of final state partons, thus affecting hadronic $p_{\perp}$-spectra. In Sec. VI A, we calculated this effect in terms of the quenching factor $Q\left(p_{\perp}\right)$. Alternatively, this quenching factor can be determined from medium-modified fragmentation functions, which we discuss now.

In the QCD-improved parton model, hadronic cross sections for high- $p_{\perp}$ hadroproduction are calculated by convoluting the perturbatively calculable hard partonic cross section $d \sigma^{q}$ and the (final state) fragmentation function $D_{h / q}\left(x, Q^{2}\right)$,

$$
d \sigma^{h}\left(z, Q^{2}\right)=\left(\frac{d \sigma^{q}}{d y}\right) d y D_{h / q}\left(x, Q^{2}\right) d x .
$$

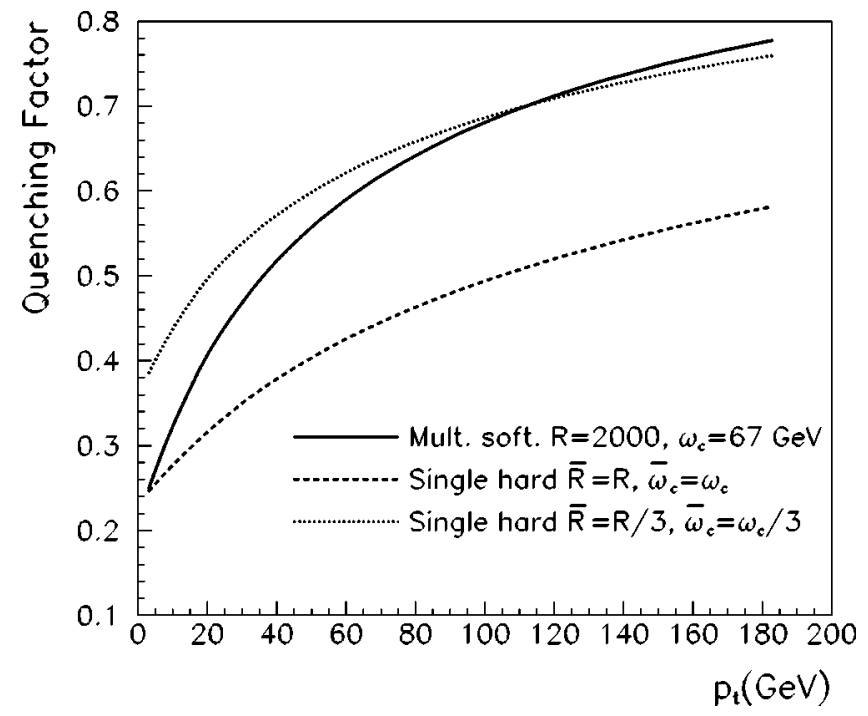

FIG. 17. Comparison of the quenching factor (6.2) calculated for $\alpha_{s}=1 / 3$ and effective power $n=7$ in the multiple soft, single hard, and rescaled single hard scattering approximation.

For notational simplicity, we do not denote the additional convolution of $d \sigma^{q}$ with the (initial state) parton distributions. The leading hadron $h$ carries an energy fraction $z$ $=E_{h} / Q$ of the total virtuality of the partonic collision, which is a fraction $x=E_{h} / E_{q}$ of the energy of its parent parton. The parent parton carries the energy fraction $y=E_{q} / Q$.

If the parent parton loses with probability $P(\epsilon)$ an additional energy fraction $\epsilon=\Delta E / E_{q}$ prior to hadronization, then the leading hadron is a fragment of a parton with lower energy $(1-\epsilon) E_{q}$; thus, it carries a larger fraction $x /(1-\epsilon)$ of the initial parton energy. The inclusion of this effect amounts to replacing the fragmentation function $D_{h / q}\left(x, Q^{2}\right)$ in Eq. (6.3) by the medium-modified fragmentation function $[24,25]$

$$
D_{h / q}^{(\mathrm{med})}\left(x, Q^{2}\right)=\int_{0}^{1} d \epsilon P(\epsilon) \frac{1}{1-\epsilon} D_{h / q}\left(\frac{x}{1-\epsilon}, Q^{2}\right) .
$$

To calculate Eq. (6.4), we use the recent LO fragmentation functions of Kniehl, Kramer, and Pötter [26] (KKP). These improve over previously available parametrizations [27]. However, the KKP parametrization still shows significant uncertainties in the large- $x$ region relevant for hadronic $p_{\perp}$ spectra [28]. For alternative approaches towards mediummodified fragmentation functions, see Refs. [29,30].

We have calculated the medium-modified $q \rightarrow \pi$ and $g$ $\rightarrow \pi$ fragmentation functions (6.4), using the quenching weights in the multiple soft (Fig. 18) and single hard (Fig. 19) scattering approximation. The energy of the parent parton is set to the virtuality of the hard process [11] $E_{q} \sim Q$. The medium-induced fragmentation functions decrease with increasing density of the medium since the probability of a parton of initial energy $E_{q}$ to fragment into a hadron of large energy $x E_{q}$ decreases with increasing parton energy loss. They should be trusted for sufficiently large momentum fractions ( $x>0.1$ say) only. The reason is that the hadronized 


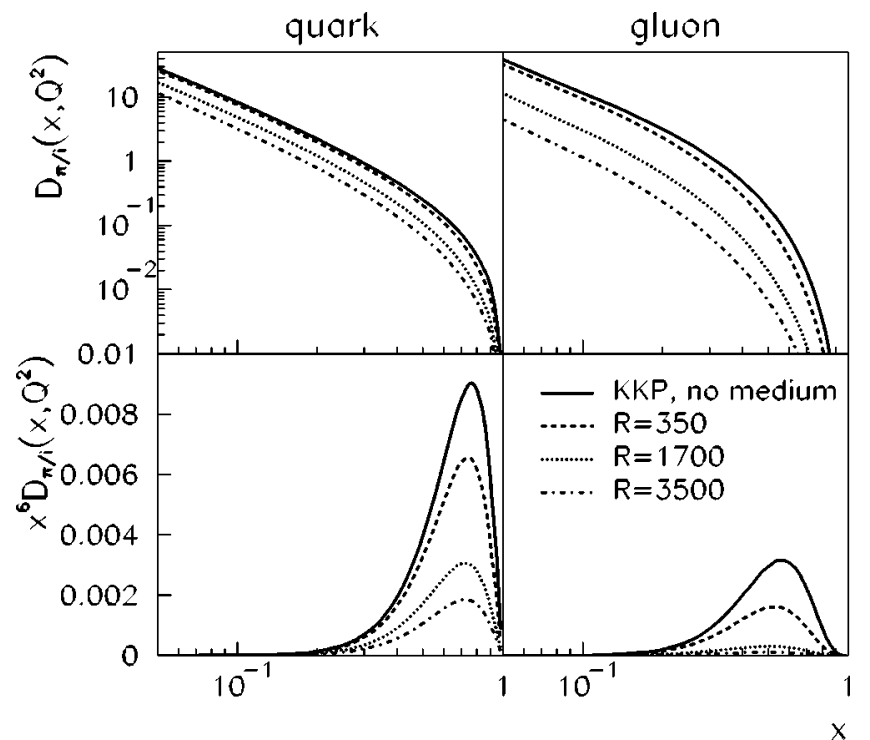

FIG. 18. The medium-modified fragmentation function (6.4) for $Q^{2}=(10 \mathrm{GeV})^{2}$ calculated in the multiple soft scattering approximation for a medium of length $L=6 \mathrm{fm}$.

remnants of the medium-induced soft radiation are not included in the definition of Eq. (6.4). These remnants are soft-they can be expected to give an additional contribution in the region $x<0.1$. The neglect of these remnants in Eq. (6.4) implies that the normalization of $D_{h / q}^{(\mathrm{med})}\left(x, Q^{2}\right)$ is a factor $\int d \epsilon \epsilon P(\epsilon)$ too small,

$$
\int_{0}^{1} d x x D_{h / q}^{(\mathrm{med})}(x) \simeq \int_{0}^{1} d x x D_{h / q}(x) \int d \epsilon(1-\epsilon) P(\epsilon) .
$$

For the suppression of high- $p_{\perp}$ hadronic spectra, this normalization error is unimportant since the main contribution comes from the region of larger $x$.

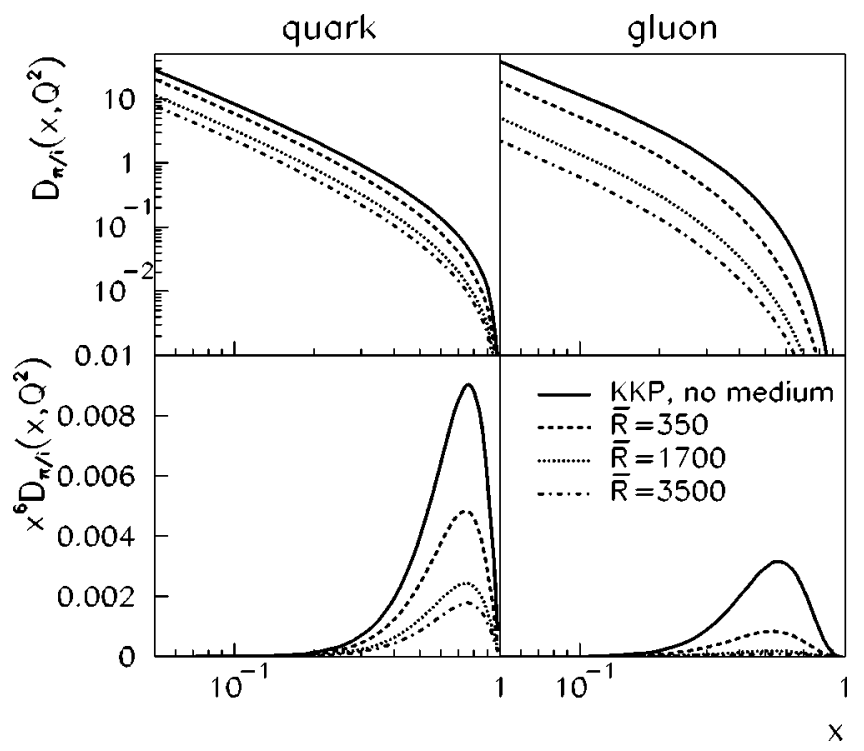

FIG. 19. The medium-modified fragmentation function (6.4) for $Q^{2}=(10 \mathrm{GeV})^{2}$ calculated in the single hard scattering approximation for a medium of length $L=6 \mathrm{fm}$.
To estimate the corresponding suppression of high- $p_{\perp}$ hadronic spectra, we exploit that the fragmentation function in Eq. (6.3) is weighed by the partonic cross section $d \sigma^{q} / d p_{\perp}^{2}$ (we work in the frame in which the total energy of the produced parton goes transverse to the beam). In the kinematic range relevant for RHIC $\left(\sqrt{s}=200 \mathrm{GeV}\right.$ and $p_{\perp}$ $\sim 10 \mathrm{GeV})$, one finds [31] $d \sigma^{q} / d p_{\perp}^{2} \sim 1 / p_{\perp}^{n\left(\sqrt{s}, p_{\perp}\right)}$ with $n\left(\sqrt{s}, p_{\perp}\right) \sim 6$. Thus, Eq. (6.3) effectively tests $x^{n\left(\sqrt{s}, p_{\perp}\right)} D_{h / q}^{(\text {med })}\left(x, Q^{2}\right)$. The suppression factor

$$
R_{f f}\left(p_{\perp}\right)=\left.\frac{x_{\max }^{6} D_{h / q}^{(\operatorname{med})}\left(x_{\max }, p_{\perp}^{2}\right)}{x_{\max }^{6} D_{h / q}\left(x_{\max }, p_{\perp}^{2}\right)}\right|_{p_{\perp}=x_{\max } E_{q}}
$$

provides a simple estimate of the reduction of hadronic $p_{\perp}$ spectra. In Eq. (6.6), $x_{\max }$ denotes the maximum of $x^{n\left(\sqrt{s}, p_{\perp}\right)} D_{h / q}^{\text {(med) }}\left(x, Q^{2}\right)$ and corresponds to the most likely energy fraction $p_{\perp}=x_{\max } E_{q}$ of the leading hadron. The suppression factor can be read off easily from the lower rows of Figs. 18 and 19. We now compare this suppression factor to the quenching factor $Q\left(p_{\perp}\right)$ in Eq. (6.2).

\section{The nuclear modification factor}

Experimental situation. Published data for $\mathrm{Au}+\mathrm{Au}$ collisions at $\sqrt{s_{N N}}=130 \mathrm{GeV}$ show for $p_{\perp}<6 \mathrm{GeV}$ a suppression of neutral pion [32] and charged hadron [32,33] transverse momentum spectra if compared to spectra in $p+p$ collisions rescaled by the number of binary collisions. This suppression is most pronounced (up to a factor $\sim 5$ ) in central $\mathrm{Au}+\mathrm{Au}$ collisions and smoothly approaches the binary scaling case with decreasing centrality. Within error bars, the suppression factors of $\pi^{0}$ and charged hadron spectra agree, though central values for the suppression of $\pi^{0}$ production are slightly lower [32]. In addition, a maximal azimuthal anisotropy $v_{2}\left(p_{\perp}\right)$ of hadroproduction is found to persist up to the highest transverse momentum [34-36]. These data indicate the importance of final state medium effects up to $p_{\perp}<6 \mathrm{GeV}$.

Preliminary data shown at the Quark Matter 2002 conference confirm these findings for $\mathrm{Au}+\mathrm{Au}$ collisions at $\sqrt{s_{N N}}$ $=200 \mathrm{GeV}$; they extend many observations up to $p_{\perp}$ $\sim 10 \mathrm{GeV}$. In particular, data for the nuclear modification factor show an approximately constant maximal suppression within $6<p_{\perp}<12 \mathrm{GeV}$ for charged hadrons [37-39] and up to $p_{\perp}<8 \mathrm{GeV}$ for $\pi^{0}$ spectra $[40,41]$. The azimuthal anisotropy $v_{2}\left(p_{\perp}\right)$ of charged hadrons stays close to maximal up to $p_{\perp}<10 \mathrm{GeV}$ [42]. Moreover, the disappearance of back-toback high- $p_{\perp}$ hadron correlations [43-45] provides an additional indication that final state medium effects play a decisive role in hadroproduction up to $p_{\perp} \sim 10 \mathrm{GeV}$.

Theoretical situation. Parton energy loss has been proposed to account for the small nuclear modification factor $[17,21,46]$, the azimuthal anisotropy $[19,47-50]$ and the disappearance of dijets [51,52]. Quantitative studies indicate, however, that in the kinematic regime relevant for RHIC $\left(p_{\perp}<12 \mathrm{GeV}\right), p_{\perp}$ broadening [52,21], shadowing [46,21], formation time [17], and possibly other effects contribute to the high- $p_{\perp}$ nuclear modification as well. Indeed, models 


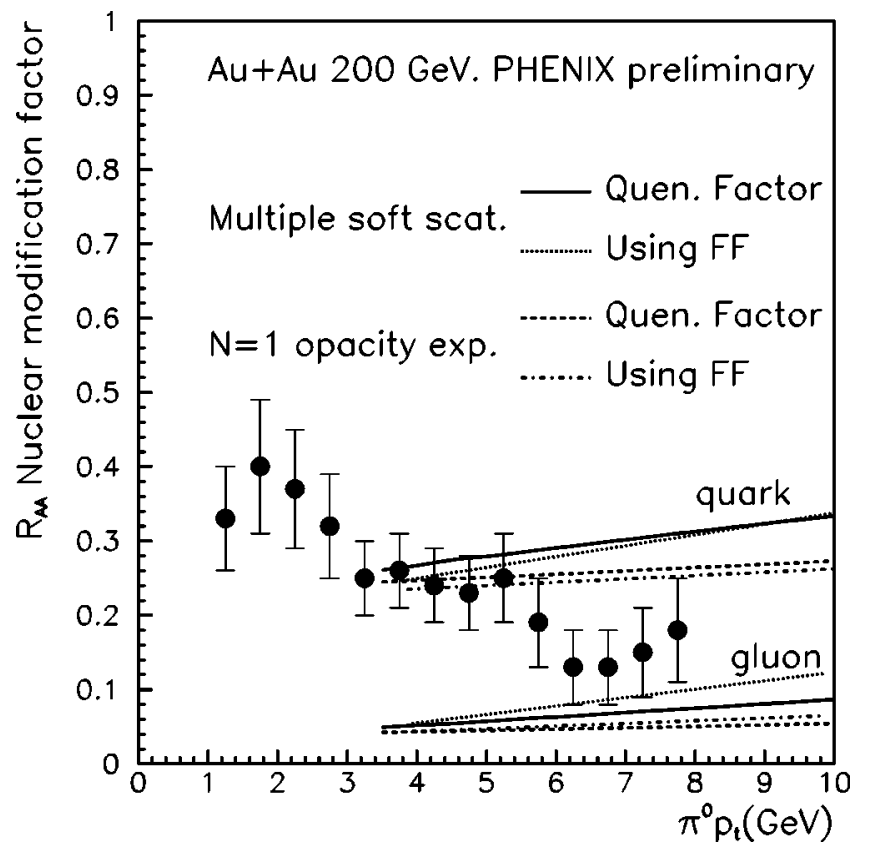

FIG. 20. The nuclear modification factor for $\pi^{0}$ production $[40,41]$ compared to model calculations involving parton energy loss only. The lines show the quenching factor (6.2) with effective power $n=7$, and the suppression factor (6.6) calculated from medium-modified fragmentation functions. They are given in the limiting cases where all parent partons are either quarks (upper lines) or gluons (lower lines). Calculations in the multiple soft scattering approximation use $R=2000, \omega_{c}=67.5 \mathrm{GeV}$, corresponding to $\hat{q}=0.75 \mathrm{GeV}^{2} / \mathrm{fm}$ and $L=6 \mathrm{fm}$. In the single hard scattering approximation, we use $\bar{R}=R, \bar{\omega}_{c}=\omega_{c}$.

have been proposed which account for hadronic quenching without taking recourse to parton energy loss. Instead, these models invoke string percolation [53], small hadronization time arguments [54], saturation physics [55], the dominance of parton recombination over parton fragmentation [56], or initial state formation time arguments [57]. The consistency and applicability of these models is currently under debate. For the $p_{\perp}$ range accessible to RHIC, the competing hadronic effects may make it difficult to disentangle quantitatively the contribution of parton energy loss from the measured hadronic suppression pattern. The transverse phase space accessible to LHC $\left(E_{\perp}<200 \mathrm{GeV}\right)$ may turn out to be a qualitative advantage with this respect.

Model comparison. In Fig. 20, we compare the two definitions (6.2) and (6.6) of the quenching factor to the nuclear modification factor measured by the PHENIX Collaboration in the $\pi^{0}$ spectra $[40,41]$ of central $\mathrm{Au}+\mathrm{Au}$ collisions at $\sqrt{s_{N N}}=200 \mathrm{GeV}$. We do not include the nuclear modification factor for charged hadrons [37-39] in Fig. 20, since charged hadrons are likely to be dominated at high $p_{\perp}$ by baryons whose production mechanism may involve additional nonperturbative effects [58].

Our comparison makes several simplifying assumptions which can be improved in further studies. (i) We do not model a realistic space-time geometry of the collision. Instead, we work for a fixed in-medium path length $L$
$=6 \mathrm{fm}$. As a consequence, we do not compare to the centrality dependence of the nuclear modification factor for which a realistic distribution of in-medium path lengths and their impact parameter dependence is needed. (ii) We do not calculate the hard partonic matrix elements entering, e.g., Eq. (6.3). Instead, we determine the quenching factors directly from Eqs. (6.2) and (6.6). As a consequence, we do not know the $p_{\perp}$-dependent fractions of hard quarks and of hard gluons which fragment into a leading $\pi^{0}$. Since parton energy loss is different for quarks and gluons, we plot in Fig. 20 the limits for which all parent partons are quarks or gluons, respectively. The realistic curve lies in between these limits. Since the ratio of parent quarks over parent gluons increases with $p_{\perp}$, the $p_{\perp}$ dependence of the realistic curve will be slightly steeper than the limiting cases presented in Fig. 20.

From Fig. 20, we can draw several conclusions. First, the two definitions of quenching factors in terms of hadronic spectra (6.2) and in terms of fragmentation functions (6.6) lead to quantitatively comparable results. Second, the multiple soft and single hard scattering approximations for parton energy loss lead to quantitatively comparable results. The slight variations in $p_{\perp}$ slope should be regarded as theoretical uncertainties in approximating Eq. (2.1) and do not give any preference to either approximation scheme. Third, a calculation based on partonic energy loss only can reproduce the magnitude of the observed nuclear modification factor. Moreover, it results in a very shallow $p_{\perp}$ dependence which seems consistent with the current quality of experimental data. For an interpretation of the model parameters used in Fig. 20, we use for a Bjorken scaling expansion $(\alpha=1)$ the relation [11,19] between the kinematic constraint $R$ and the initially produced gluon density

$$
R=\frac{L^{2}}{R_{A}^{2}} \frac{d N^{g}}{d y}
$$

where $R_{A}$ denotes the nuclear radius. The extracted value $d N^{g} / d y \simeq 2000$ is approximately a factor 2 larger than a previous estimate [19] based on data from elliptic flow. Given the theoretical uncertainties of parton energy loss calculations below $p_{\perp}<10 \mathrm{GeV}$, this factor 2 mismatch constitutes no inconsistency.

\section{CONCLUSION}

In recent years, phenomenological applications of medium-induced parton energy loss were based mainly on two different approximations of the medium-induced gluon energy distribution: the multiple soft BDMPS-Zakharov (BDMPSZ) scattering approximation and the opacity approximation. It remained unclear, however, to what extent these approximations differ. Here, we have studied in both approximations the medium-induced energy distributions (Sec. II), the corresponding quenching weights (Sec. III), and the extension of these calculations to dynamically expanding collision regions (Sec. IV), and to the angular dependence of the medium-induced radiation pattern (Sec. V). The single hard scattering approximation is dominated by the hard region $\omega>\bar{\omega}_{c}$ of the gluon energy distribution while the mul- 
tiple soft scattering approximation is dominated by the soft region $\omega<\omega_{c}$. Despite this difference, both approximations lead to quantitatively comparable results if comparable sets of model parameters are used. Numerically, we determine the correspondence

$$
\omega_{c} \simeq 3 \bar{\omega}_{c}, \quad R \simeq 3 \bar{R},
$$

which relates the BDMPS transport coefficient $\hat{q}$ and the Debye screening mass $\mu^{2}$ via an opacity $n_{0} L \sim 3$, see Eq. (3.12). Deviations from Eq. (7.1) can be understood in terms of kinematic constraints on the hard part of the gluon energy distribution (see discussion of Fig. 17).

The main result of this paper is the calculation of quenching weights in Sec. III. We explained how to calculate from these quenching weights the nuclear modification of hadronic spectra. There are indications that the interpretation of RHIC data on hadronic quenching requires additional physics effects beyond the parton energy loss (see discussion in Sec. VI C). However, to discriminate energy loss contributions from these additional effects, a quantitatively reliable discussion of the current theoretical calculations of parton energy loss is needed. We hope that the CPU-inexpensive subroutine for quenching weights which accompanies this paper will prove a valuable tool to this end. Also, this routine can be used to explore observable consequences of parton energy loss in nucleus-nucleus collisions at LHC or for processes in cold nuclear matter.

\section{ACKNOWLEDGMENTS}

We thank Francois Arleo, Nestor Armesto, Rolf Baier, Yuri Dokshitzer, Kari Eskola, Alex Kovner, Andreas Morsch, Jürgen Schukraft, and Boris Tomasik for helpful discussions. In particular, we thank Rolf Baier for suggesting the argument leading to Eqs. (2.12) and (2.13). C.A.S. is supported by a Marie Curie Fellowship of the European Community program TMR (Training and Mobility of Researchers) under Contract No. HPMF-CT-2000-01025.

\section{APPENDIX A: THE BDMPS-LIMIT OF THE ENERGY DISTRIBUTION (2.1)}

Here, we establish that the $R \rightarrow \infty$ limit of the gluon energy distribution (2.1) coincides with the BDMPS result (2.9). Using the saddle point approximation (2.3), the energy distribution (2.1) can be written in the form given in Eqs. (A11) and (A12) of Ref. [6]. Integrated over transverse momentum $0<k_{\perp}<\chi \omega$, one finds

$$
\omega \frac{d I}{d \omega}=\frac{\alpha_{s} C_{F}}{\pi}\left(I_{4}+I_{5}\right)
$$

$$
\begin{aligned}
I_{4}= & \frac{1}{4 \omega^{2}} 2 \operatorname{Re} \int_{0}^{L} d y_{l} \int_{y_{l}}^{L} d \bar{y}_{l} 16 A_{4}^{2} \\
& \times\left[\left(1-\frac{i A_{4} B_{4}(\chi \omega)^{2}}{4\left(D_{4}-i A_{4} B_{4}\right)^{2}}\right)\right. \\
& \left.\times \exp \left\{-\frac{(\chi \omega)^{2}}{4\left(D_{4}-i A_{4} B_{4}\right)^{2}}\right\}-1\right], \\
I_{5}= & \frac{1}{\omega} \operatorname{Re} \int_{0}^{L} d y_{l} \frac{4 A_{5}}{B_{5}}\left[\exp \left\{-\frac{i(\chi \omega)^{2}}{4 A_{5} B_{5}}\right\}-1\right],
\end{aligned}
$$

where

$$
\begin{aligned}
& A_{4}=\frac{\omega \Omega}{2 \sin \left[\Omega\left(\bar{y}_{L}-y_{L}\right)\right]}, \quad B_{4}=\cos \left[\Omega\left(\bar{y}_{L}-y_{L}\right)\right], \\
& D_{4}=\frac{1}{2} n_{0} C\left(L-\bar{y}_{L}\right), \\
& A_{5}=\frac{\omega \Omega}{2 \sin \left(\Omega y_{L}\right)}, \quad B_{5}=\cos \left(\Omega y_{L}\right),
\end{aligned}
$$

and

$$
\Omega=(1+i) \sqrt{\frac{\hat{q}}{4 \omega}} .
$$

The limit $R \rightarrow \infty$ is obtained by taking $\chi \rightarrow \infty$ in Eqs. (A2) and (A3):

$$
\begin{aligned}
\lim _{R \rightarrow \infty} I_{4} & =-2 \operatorname{Re} \int_{0}^{L} d y_{l} \int_{y_{l}}^{L} d \bar{y}_{l} \frac{\Omega^{2}}{\sin ^{2}\left[\Omega\left(\bar{y}_{l}-y_{l}\right)\right]} \\
& =-\operatorname{Re} \int_{0}^{L} d y_{l} \int_{0}^{L} d \bar{y}_{l} \frac{\Omega^{2}}{\sin ^{2}\left[\Omega\left(\bar{y}_{l}-y_{l}\right)\right]} \\
& =2 \operatorname{Re} \int_{0}^{L} d y_{l} \Omega \frac{\cos \left(\Omega y_{l}\right)}{\sin \left(\Omega y_{l}\right)}, \\
\lim _{R \rightarrow \infty} I_{5} & =-2 \operatorname{Re} \int_{0}^{L} d y_{l} \frac{\Omega}{\sin \left(\Omega y_{l}\right) \cos \left(\Omega y_{l}\right)} .
\end{aligned}
$$

Both integrals are logarithmically divergent but this divergence cancels in the sum

$$
\lim _{R \rightarrow \infty}\left(I_{4}+I_{5}\right)=2 R e \ln [\cos (\Omega L)] .
$$

This coincides with the BDMPS result (2.9). 


\section{APPENDIX B: GLUON ENERGY DISTRIBUTION TO FIRST ORDER IN OPACITY}

In this appendix, we calculate the first order in opacity of the gluon energy distribution (2.1) for a Yukawa-type elastic scattering center with Debye screening mass $\mu$ :

$$
|a(\mathbf{q})|^{2}=\frac{\mu^{2}}{\pi\left(\mathbf{q}^{2}+\mu^{2}\right)^{2}} .
$$

According to Eq. (6.4) of Ref. [4], the energy distribution takes the form

$$
\begin{aligned}
\omega \frac{d I^{N=1}}{d \omega}= & \frac{\alpha_{s}}{(2 \pi)^{2}} \frac{2 C_{R}}{\omega^{2}} \int_{0}^{\chi \omega} d \mathbf{k} \int_{0}^{\infty} d \mathbf{q} \frac{\mu^{2}}{\pi\left(\mathbf{q}^{2}+\mu^{2}\right)^{2}} \\
& \times \mathbf{k} \cdot \mathbf{q} Z\left(Q, Q_{1}\right),
\end{aligned}
$$

where

$$
Q=\frac{\mathbf{k}^{2}}{2 \omega}, \quad Q_{1}=\frac{(\mathbf{k}+\mathbf{q})^{2}}{2 \omega}
$$

and

$$
\begin{aligned}
Z\left(Q, Q_{1}\right)= & \lim _{\epsilon \rightarrow 0} \operatorname{Re} \int_{\xi_{0}}^{\infty} d y \int_{y}^{\infty} d \bar{y} e^{-\epsilon y-\epsilon \bar{y}} \\
& \times \int_{y}^{\bar{y}} d \xi n_{0}\left(\frac{\xi_{0}}{\xi}\right)^{\alpha} e^{-i Q(\bar{y}-\xi)-i Q_{1}(\xi-y)} \\
\equiv & \frac{n_{0}}{Q} \bar{Z}\left(Q_{1}\right) .
\end{aligned}
$$

Irrespective of the value of the expansion parameter $\alpha$ in (B4), the expression factorizes in the form (B5). In order to simplify Eq. (B2), we shift the integration variables by $\mathbf{q}$ $\rightarrow \mathbf{q}-\mathbf{k}, \mathbf{k} \rightarrow \mathbf{k} \sqrt{2 \omega / L}$ and $\mathbf{q} \rightarrow \mathbf{q} \sqrt{2 \omega / L}$. This leads to

$$
\begin{aligned}
\omega \frac{d I^{N=1}}{d \omega}= & 4 \frac{\alpha_{s} C_{R}}{\pi} \int_{0}^{\kappa} d k \int_{0}^{2 \pi} d \varphi \int_{0}^{\infty} q d q \frac{\partial}{\partial k} \\
& \times\left(\frac{1}{k^{2}+2 k q \cos \varphi+q^{2}+\gamma}\right)\left(\frac{\gamma}{2 \pi}\right) \frac{n_{0}}{L} \bar{Z}\left(q^{2} / L\right),
\end{aligned}
$$

where

$$
\gamma=\frac{\mu^{2} L}{2 \omega}, \quad \kappa=\chi \sqrt{\frac{\omega L}{2}}=\sqrt{\frac{\bar{R}}{2 \gamma}} .
$$

The $\varphi$ - and $k$-integration in Eq. (B6) can be done analytically,

$$
\begin{aligned}
\omega \frac{d I^{N=1}}{d \omega}= & 4 \frac{\alpha_{s} C_{R}}{\pi} \frac{n_{0}}{L} \int_{0}^{\infty} q d q \bar{Z}\left(q^{2} / L\right) \\
& \times\left(\frac{\gamma}{\sqrt{\left(\kappa^{2}+q^{2}+\gamma\right)^{2}-4 \kappa^{2} q^{2}}}-\frac{\gamma}{q^{2}+\gamma}\right) .
\end{aligned}
$$

(1) For the static case $\alpha=0$, the phase factor (B5) reads

$$
\bar{Z}_{\alpha=0}\left(Q_{1}\right)=\frac{-L Q_{1}+\sin \left(L Q_{1}\right)}{Q_{1}^{2}}
$$

and

$$
\begin{aligned}
\omega \frac{d I_{\alpha=0}^{N=1}}{d \omega}= & 4 \frac{\alpha_{s} C_{R}}{\pi}\left(n_{0} L\right) \gamma \int_{0}^{\infty} q d q \frac{q^{2}-\sin \left(q^{2}\right)}{q^{4}} \\
& \times\left(\frac{1}{q^{2}+\gamma}-\frac{1}{\sqrt{\left(\kappa^{2}+q^{2}+\gamma\right)^{2}-4 \kappa^{2} q^{2}}}\right) .
\end{aligned}
$$

Substituting $r=q^{2}$, we find Eq. (2.19).

(2) For the Bjorken scaling case $\alpha=1$, the phase (B5) reads

$$
\begin{aligned}
\bar{Z}_{\alpha=1}\left(Q_{1}\right)= & \frac{\xi_{0}}{Q_{1}} \operatorname{Re}\left[e ^ { i Q _ { 1 } \xi _ { 0 } } \left(\operatorname{Ei}\left[-i Q_{1}\left(L+\xi_{0}\right)\right]\right.\right. \\
& \left.\left.-\operatorname{Ei}\left[-i Q_{1} \xi_{0}\right]\right)+\ln \frac{\xi_{0}}{L+\xi_{0}}\right] \\
= & \frac{\xi_{0}}{Q_{1}} \operatorname{Re}\left[\operatorname{Ei}\left[-i Q_{1} L\right]-\ln \left[-i Q_{1} L\right]\right. \\
& \left.-\gamma_{E}+O\left(\xi_{0} / L\right)\right] \\
= & \frac{\xi_{0}}{Q_{1}} \operatorname{Re}\left[\int_{0}^{-i Q_{1} L} d t \frac{e^{-t}-1}{t}+O\left(\xi_{0} / L\right)\right] .
\end{aligned}
$$

Here, $\gamma_{E}=0.577 \cdots$ is the Euler constant and the exponential integral function Ei is defined in the text following Eq. (4.3). Corrections of order $O\left(\xi_{0} / L\right)$ can be ignored since the time of production $\xi_{0}$ is much smaller than the in-medium path length $L$. With this approximation, one has

$$
\begin{aligned}
\omega \frac{d I_{\alpha=1}^{N=1}}{d \omega}= & 4 \frac{\alpha_{s} C_{R}}{\pi}\left(n_{0} \xi_{0}\right) \int_{0}^{\infty} \frac{q d q}{q^{2}} \operatorname{Re}\left[-\operatorname{Ei}\left[-i q^{2}\right]\right. \\
& \left.+\ln \left[-i q^{2}\right]+\gamma_{E}\right] \\
& \times\left(\frac{\gamma}{q^{2}+\gamma}-\frac{\gamma}{\sqrt{\left(\kappa^{2}+q^{2}+\gamma\right)^{2}-4 \kappa^{2} q^{2}}}\right) .
\end{aligned}
$$

Substituting $r=q^{2}$, we find Eq. (4.3). 


\section{APPENDIX C: THE DIPOLE APPROXIMATION FOR AN EXPANDING MEDIUM}

In this appendix, we follow Ref. [20] in giving explicit expressions for the path integral (2.14) in the dipole approximation

$$
\begin{aligned}
& \mathcal{K}\left(\mathbf{r}_{1}, y_{1} ; \mathbf{r}_{2}, y_{2} \mid \omega\right) \\
& =\int \mathcal{D} \mathbf{r} \exp \left[i \frac{\omega}{2} \int_{y_{1}}^{y_{2}} d \xi\left(\dot{\mathbf{r}}^{2}-\frac{\Omega_{\alpha}^{2}\left(\xi_{0}\right)}{\xi^{\alpha}} \mathbf{r}^{2}\right)\right]
\end{aligned}
$$

Equation $(\mathrm{C} 1)$ is the path integral of a 2-dimensional harmonic oscillator with time-dependent imaginary frequency

$$
\frac{\Omega_{\alpha}^{2}\left(\xi_{0}\right)}{\xi^{\alpha}}=\frac{\hat{q}(\xi)}{i 2 \omega}=-i \frac{\hat{q}_{0}}{2 \omega}\left(\frac{\xi_{0}}{\xi}\right)^{\alpha}
$$

and mass $\omega$. The solution of Eq. (C1) can be written in the form [20]

$$
\mathcal{K}\left(\mathbf{r}_{1}, y_{1} ; \mathbf{r}_{2}, y_{2} \mid \omega\right)=\frac{\omega}{2 \pi i D\left(y_{1}, y_{2}\right)} \exp \left[i S_{\mathrm{cl}}\left(\mathbf{r}_{1}, y_{1} ; \mathbf{r}_{2}, y_{2}\right)\right] \text {. }
$$

Here, the classical action $S_{\mathrm{cl}}$ in Eq. (C3) takes the form

$$
S_{\mathrm{cl}}\left(\mathbf{r}_{1}, y_{1} ; \mathbf{r}_{2}, y_{2}\right)=\left.\frac{\omega}{2}\left[\mathbf{r}_{\mathrm{cl}}(\xi) \cdot \frac{d}{d \xi} \mathbf{r}_{\mathrm{cl}}(\xi)\right]\right|_{y_{2}} ^{y_{1}}
$$

where the classical path $\mathbf{r}_{\mathrm{cl}}(\xi)$ satisfies the homogeneous differential equation

$$
\left[\frac{d^{2}}{d \xi^{2}}-\frac{\Omega_{\alpha}^{2}\left(\xi_{0}\right)}{\xi^{\alpha}}\right] \mathbf{r}_{\mathrm{cl}}(\xi)=0
$$

with initial conditions

$$
\mathbf{r}_{\mathrm{cl}}\left(y_{1}\right)=\mathbf{r}_{1} \quad \text { and } \quad \mathbf{r}_{\mathrm{cl}}\left(y_{2}\right)=\mathbf{r}_{2} \text {. }
$$

The fluctuation determinant $D\left(\xi, \xi^{\prime}\right)$ in Eq. (C3) satisfies

$$
\left[\frac{d^{2}}{d \xi^{2}}-\frac{\Omega_{\alpha}^{2}\left(\xi_{0}\right)}{\xi^{\alpha}}\right] D\left(\xi, \xi^{\prime}\right)=0
$$

with initial conditions

$$
D(\xi, \xi)=0 \quad \text { and }\left.\frac{d}{d \xi} D\left(\xi, \xi^{\prime}\right)\right|_{\xi=\xi^{\prime}}=1 .
$$

In practice, $D\left(\xi, \xi^{\prime}\right)$ is found by combining the two independent (scalar) solutions $f_{1}, f_{2}$ of Eq. (C5),

$$
D\left(\xi, \xi^{\prime}\right)=\mathcal{N}\left[f_{1}(\xi) f_{2}\left(\xi^{\prime}\right)-f_{2}(\xi) f_{1}\left(\xi^{\prime}\right)\right]
$$

and fixing the norm $\mathcal{N}$ by the initial condition $(\mathrm{C} 8)$. The solution of Eq. (C1) can be written in terms of $D\left(\xi, \xi^{\prime}\right)$ and two $\xi$ - and $\xi^{\prime}$-dependent variables $c_{1}, c_{2}$,

$$
\begin{aligned}
\mathcal{K}\left(\mathbf{r}_{1}, y_{1} ; \mathbf{r}_{2}, y_{2} \mid \omega\right)= & \frac{i \omega}{2 \pi D\left(y_{1}, y_{2}\right)} \exp \left[-\frac{-i \omega}{2 D\left(y_{1}, y_{2}\right)}\right. \\
& \left.\times\left(c_{1} \mathbf{r}_{1}^{2}+c_{2} \mathbf{r} 2^{2}-2 \mathbf{r}_{1} \cdot \mathbf{r}_{2}\right)\right] .
\end{aligned}
$$

We consider three cases.

(1) The case $\alpha<2$. For this case, explicit expressions for Eq. (C10) are given in Appendix B of Ref. [20]. The two independent solutions of the homogeneous differential equation (C5) are

$$
\begin{aligned}
& f_{1}(\xi)=\sqrt{\xi} I_{\nu}\left[2 \nu \Omega_{\alpha}\left(\xi_{0}\right) \xi^{1 / 2 \nu}\right] \\
& f_{2}(\xi)=\sqrt{\xi} K_{\nu}\left[2 \nu \Omega_{\alpha}\left(\xi_{0}\right) \xi^{1 / 2 \nu}\right]
\end{aligned}
$$

where $I_{\nu}$ and $K_{\nu}$ are modified Bessel functions with argument

$$
\nu=\frac{1}{2-\alpha}
$$

In terms of the variable [use $\Omega_{\alpha}\left(\xi_{0}\right)=\sqrt{-i\left(\hat{q}_{0} / 2 \omega\right) \xi_{0}^{\alpha}}$ ]

$$
z(\xi)=2 \nu \Omega_{\alpha}\left(\xi_{0}\right) \xi^{1 / 2 \nu}
$$

the solution $(\mathrm{C} 10)$ is given by [20] [we use $z \equiv z(\xi), z^{\prime}$ $\left.\equiv z\left(\xi^{\prime}\right)\right]$

$$
\begin{aligned}
D\left(\xi, \xi^{\prime}\right)= & \frac{2 \nu}{\left(2 \nu \Omega_{\alpha}\left(\xi_{0}\right)\right)^{2 \nu}}\left(z z^{\prime}\right)^{\nu}\left[I_{\nu}(z) K_{\nu}\left(z^{\prime}\right)\right. \\
& \left.-K_{\nu}(z) I_{\nu}\left(z^{\prime}\right)\right] \\
c_{1}= & z\left(\frac{z^{\prime}}{z}\right)^{\nu}\left[I_{\nu-1}(z) K_{\nu}\left(z^{\prime}\right)+K_{\nu-1}(z) I_{\nu}\left(z^{\prime}\right)\right]
\end{aligned}
$$

$$
c_{2}=z^{\prime}\left(\frac{z}{z^{\prime}}\right)^{\nu}\left[K_{\nu}(z) I_{\nu-1}\left(z^{\prime}\right)+I_{\nu}(z) K_{\nu-1}\left(z^{\prime}\right)\right] \text {. }
$$

(2) The case $\alpha=2$. In this case, the two independent solutions of the homogeneous differential equation (C5) are $f_{1}(\xi)=\xi^{(1 / 2)(1-A)}$ and $f_{2}(\xi)=\xi^{(1 / 2)(1+A)}$ where $A$ $=\sqrt{1+4 \Omega_{\alpha=2}^{2}\left(\xi_{0}\right)}$. From this, one finds

$$
\begin{aligned}
D\left(\xi, \xi^{\prime}\right) & =\frac{1}{A}\left(\xi \xi^{\prime}\right)^{(1 / 2)(1-A)}\left(\xi^{A}-\xi^{\prime A}\right), \\
c_{1} & =\frac{1+A}{A}\left(\frac{\xi}{\xi^{\prime}}\right)^{-(1 / 2)(1-A)}-\frac{1-A}{A}\left(\frac{\xi^{\prime}}{\xi}\right)^{(1 / 2)(1+A)} \\
c_{2} & =\frac{1+A}{A}\left(\frac{\xi^{\prime}}{\xi}\right)^{-(1 / 2)(1-A)}-\frac{1-A}{A}\left(\frac{\xi}{\xi^{\prime}}\right)^{(1 / 2)(1+A)}
\end{aligned}
$$


(3) The case $\alpha>2$. In this case, the solution (C15)-(C17) has the argument

$$
z(\xi)=2|\nu| \Omega_{\alpha}\left(\xi_{0}\right) \xi^{1 / 2 \nu} .
$$

Modified Bessel functions with negative index can be avoided with the help of the identities $K_{\nu}(z)=K_{-\nu}(z)$ and $I_{\nu}(z)-I_{-\nu}(z)=-2[\sin (\nu \pi) / \pi] K_{\nu}(z)$.

$$
\begin{aligned}
D\left(\xi, \xi^{\prime}\right)= & 2 \nu \sqrt{\xi \xi^{\prime}}\left[I_{\nu}(z) K_{\nu}\left(z^{\prime}\right)-K_{\nu}(z) I_{\nu}\left(z^{\prime}\right)\right], \\
c_{1}= & 2|\nu| \sqrt{\frac{\xi^{\prime}}{\xi}} \xi^{1 / 2 \nu} \Omega_{\alpha}\left(\xi_{0}\right)\left[I_{\nu-1}(z) K_{\nu}\left(z^{\prime}\right)\right. \\
& \left.+K_{\nu-1}(z) I_{\nu}\left(z^{\prime}\right)\right], \\
c_{2}= & 2|\nu| \sqrt{\frac{\xi}{\xi^{\prime}}} \xi^{\prime 1 / 2 \nu} \Omega_{\alpha}\left(\xi_{0}\right)\left[K_{\nu}(z) I_{\nu-1}\left(z^{\prime}\right)\right. \\
& \left.+I_{\nu}(z) K_{\nu-1}\left(z^{\prime}\right)\right] .
\end{aligned}
$$

With the solution (C10), the radiation spectrum can be written as the sum of three contributions [6]

$$
\frac{d I^{(\mathrm{tot})}}{d \omega}=\frac{1}{\omega} \frac{d \sigma}{d \omega}=\frac{\alpha_{s}}{\pi^{2}} C_{F}\left(I_{4}+I_{5}+I_{6}\right)=\frac{d I^{(\mathrm{vac})}}{d \omega}+\frac{d I}{d \omega} .
$$

Here, $I_{6}=1 / k_{\perp}^{2}$ is the medium-independent vacuum gluon energy distribution. $I_{4}$ and $I_{5}$ determine the medium-induced part $d I / d \omega$ studied in this paper. They can be computed in the dipole approximation

$$
\begin{aligned}
I_{4}= & \frac{1}{4 \omega^{2}} 2 \operatorname{Re} \int_{\xi_{0}}^{L+\xi_{0}} d y_{l} \int_{y_{l}}^{L+\xi_{0}} d \bar{y}_{l}\left(\frac{-4 A_{4}^{2} \bar{D}_{4}}{\left(\bar{D}_{4}-i A_{4} B_{4}\right)^{2}}\right. \\
& \left.+\frac{i A_{4}^{3} B_{4} \mathbf{k}_{\perp}^{2}}{\left(\bar{D}_{4}-i A_{4} B_{4}\right)^{3}}\right) \exp \left[-\frac{\mathbf{k}_{\perp}^{2}}{4\left(\bar{D}_{4}-i A_{4} B_{4}\right)}\right], \\
I_{5}= & \frac{1}{\omega} \operatorname{Re} \int_{\xi_{0}}^{L+\xi_{0}} d y_{l} \frac{-i}{B_{5}^{2}} \exp \left[-i \frac{\mathbf{k}_{\perp}^{2}}{4 A_{5} B_{5}}\right],
\end{aligned}
$$

where

$$
\begin{aligned}
& A_{4}=\frac{\omega}{2 D\left(\bar{y}_{l}, y_{l}\right)}, \quad B_{4}=c_{1}\left(\bar{y}_{l}, y_{l}\right), \\
& \bar{D}_{4}=\frac{1}{2} \int_{\bar{y}_{l}}^{L+\xi_{0}} d \xi n(\xi) \sigma(\mathbf{r}),
\end{aligned}
$$

$$
A_{5}=\frac{\omega}{2 D\left(L+\xi_{0}, y_{l}\right)}, \quad B_{5}=c_{1}\left(L+\xi_{0}, y_{l}\right) .
$$

In the case $\alpha=0$, the functions $I_{ \pm 1 / 2}(z)$ and $K_{ \pm 1 / 2}(z)$ entering Eq. (C10) have explicit expressions in terms of exponentials. One recovers the known expressions Eqs. (A4)-(A6) for the static case $[3,6]$.
[1] R. Baier, Y.L. Dokshitzer, A.H. Mueller, and D. Schiff, J. High Energy Phys. 09, 033 (2001).

[2] R. Baier, Y.L. Dokshitzer, A.H. Mueller, S. Peigne, and D. Schiff, Nucl. Phys. B483, 291 (1997).

[3] B.G. Zakharov, JETP Lett. 63, 952 (1996).

[4] U.A. Wiedemann, Nucl. Phys. B588, 303 (2000).

[5] M. Gyulassy, P. Levai, and I. Vitev, Nucl. Phys. B594, 371 (2001).

[6] U.A. Wiedemann, Nucl. Phys. A690, 731 (2001).

[7] The expression for the gluon energy distribution derived in Eq. (4.15) of Ref. [4] and used in Ref. [6] contains an erroneous additional prefactor $C_{A}$. Equation (2.1) of the present work is the corrected result.

[8] B.G. Zakharov, Yad. Fiz. 61, 924 (1998) [Phys. At. Nucl. 61, 838 (1998)].

[9] R. Baier, Y.L. Dokshitzer, A.H. Mueller, S. Peigne, and D. Schiff, Nucl. Phys. B484, 265 (1997).

[10] R. Baier, hep-ph/0209038.

[11] C.A. Salgado and U.A. Wiedemann, Phys. Rev. Lett. 89, 092303 (2002).

[12] B.G. Zakharov, JETP Lett. 65, 615 (1997).

[13] M. Gyulassy, P. Levai, and I. Vitev, Phys. Rev. Lett. 85, 5535 (2000).
[14] B.G. Zakharov, Pisma Zh. Éksp. Teor. Fiz. 73, 55 (2001) [JETP Lett. 73, 49 (2001)].

[15] Particle Data Group, K. Hagiwara et al., Phys. Rev. D 66, 010001 (2002).

[16] See EPAPS Document No. E-PRVDAQ-67-058311 for the FORTRAN subroutine for quenching weights. A direct link to this document may be found in the online article's HTML reference section. The document may also be reached via the EPAPS homepage (http://www.aip.org/pubservs/epaps.html) or ftp.aip.org in the directory /epaps/. See the EPAPS homepage for more information.

[17] F. Arleo, J. High Energy Phys. 11, 044 (2002).

[18] K.J. Eskola, K. Kajantie, P.V. Ruuskanen, and K. Tuominen, Nucl. Phys. B570, 379 (2000).

[19] M. Gyulassy, I. Vitev, and X.N. Wang, Phys. Rev. Lett. 86, 2537 (2001).

[20] R. Baier, Y.L. Dokshitzer, A.H. Mueller, and D. Schiff, Phys. Rev. C 58, 1706 (1998).

[21] E. Wang and X.N. Wang, Phys. Rev. Lett. 89, 162301 (2002).

[22] R. Baier, Y.L. Dokshitzer, A.H. Mueller, and D. Schiff, Phys. Rev. C 64, 057902 (2001).

[23] R. Baier, Y.L. Dokshitzer, A.H. Mueller, and D. Schiff, Phys. Rev. C 60, 064902 (1999). 
[24] X.N. Wang, Z. Huang, and I. Sarcevic, Phys. Rev. Lett. 77, 231 (1996).

[25] M. Gyulassy, P. Levai, and I. Vitev, Phys. Lett. B 538, 282 (2002).

[26] B.A. Kniehl, G. Kramer, and B. Potter, Nucl. Phys. B582, 514 (2000).

[27] J. Binnewies, B.A. Kniehl, and G. Kramer, Z. Phys. C 65, 471 (1995)

[28] X.F. Zhang, G. Fai, and P. Levai, Phys. Rev. Lett. 89, 272301 (2002).

[29] X.F. Guo and X.N. Wang, Phys. Rev. Lett. 85, 3591 (2000).

[30] J.A. Osborne, E. Wang, and X.N. Wang, Phys. Rev. D 67, 094022 (2003).

[31] K.J. Eskola and H. Honkanen, Nucl. Phys. A713, 167 (2003).

[32] PHENIX Collaboration, K. Adcox et al., Phys. Rev. Lett. 88, 022301 (2002).

[33] C. Adler et al., Phys. Rev. Lett. 89, 202301 (2002).

[34] STAR Collaboration, C. Adler et al., Phys. Rev. Lett. 90, 032301 (2003).

[35] STAR Collaboration, K. Filimonov, nucl-ex/0210027.

[36] PHENIX Collaboration, N.N. Ajitanand, nucl-ex/0210007.

[37] STAR Collaboration, J.L. Klay, nucl-ex/0210026.

[38] PHENIX Collaboration, J. Jia, nucl-ex/0209029.

[39] PHOBOS Collaboration, C. Roland et al., hep-ex/0212006.

[40] PHENIX Collaboration, D. d'Enterria, hep-ex/0209051.
[41] PHENIX Collaboration, S. Mioduszewski, nucl-ex/0210021.

[42] STAR Collaboration, G.J. Kunde, nucl-ex/0211018.

[43] STAR Collaboration, C. Adler et al., Phys. Rev. Lett. 90, 082302 (2003).

[44] The STAR Collaboration, D. Hardtke, nucl-ex/0212004.

[45] PHENIX Collaboration, M. Chiu, nucl-ex/0211008.

[46] S.Y. Jeon, J. Jalilian-Marian, and I. Sarcevic, nucl-th/0208012.

[47] I. Vitev and M. Gyulassy, Phys. Rev. Lett. 89, 252301 (2002).

[48] X.N. Wang, Phys. Rev. C 63, 054902 (2001).

[49] I.P. Lokhtin and A.M. Snigirev, Eur. Phys. J. C 16, 527 (2000).

[50] M. Gyulassy, I. Vitev, X.N. Wang, and P. Huovinen, Phys. Lett. B 526, 301 (2002).

[51] B. Muller, Phys. Rev. C 67, 061901(R) (2003).

[52] T. Hirano and Y. Nara, nucl-th/0301042.

[53] M.A. Braun, F. Del Moral, and C. Pajares, Phys. Rev. C 65, 024907 (2002).

[54] K. Gallmeister, C. Greiner, and Z. Xu, Phys. Rev. C 67, 044905 (2003).

[55] D. Kharzeev, E. Levin, and L. McLerran, Phys. Lett. B 561, 93 (2003).

[56] R.J. Fries, S.A. Bass, B. Muller, and C. Nonaka, Phys. Rev. Lett. 90, 202303 (2003).

[57] R. Lietava, J. Pisut, N. Pisutova, and B. Tomasik, nucl-th/0301052.

[58] I. Vitev and M. Gyulassy, Phys. Rev. C 65, 041902 (2002). 